THEORY OF SPIN WAVES IN THE HEAVY RARE EARTH METALS 


\title{
THEORY OF SPIN WAVES IN THE \\ HEAVY RARE EARTH METALS
}

\section{By}

BYRON WAYNE SOUTHERN, B.SC.

\author{
A Thesis \\ Submitted to the School of Graduate studies \\ in Partial Fulfilment of the Requirements \\ for the Degree \\ Master of Science
}

McMaster University

December, 1970 
MASTER OF SCIENCE (1970)

(Physics)

TITLE :

AUTHOR :

SUPERVISOR :

NUMBER OF PAGES :
MCMASTER UNIVERSITY

Hamilton, Ontario

SCOPE AND CONTENTS:

A theory of spin waves for the spin structures found in the rare earth metals of hop crystal structure is described. The theory is developed for the conical spiral spin structure which contains the planar spiral, the nonplanar ferromagnet and the planar ferromagnet as special cases. Included in the Hamiltonian are isotropic and anisotropic exchange interactions, single-ion crystal field terms, and magnetoelastic terms, both of the single-ion type and the two-ion type. Equations of motion for the spin operators are linearized with the help of the random phase approximation which makes it possible to express some spin-wave interaction effects in terms of powers of the reduced magnetization. Expressions for the spin-wave energies are given for the four structures under consideration. 


\section{ACKNOWLEDGEMENTS}

I wish to thank my research supervisor, Dr. D. A. Goodings, for his valuable suggestions and assistance in this investigation.

I would also like to acknowledge an enlightening discussion of the conical spiral spin structure with T. M. Holden, B. M. Powell and A. D. B. Woods.

Financial support in the form of a fellowship from the Province of Ontario and a postgraduate scholarship from the National Research Council of Canada is gratefully acknowledged.

Many thanks to Miss C. Wivell and Mrs. U. Blake for the fast and accurate typing of this thesis. 
TABLE OF CONTENTS

Page

CHAPTER 1 INTRODUCTION

1.1 Magnetic Interactions 1

1.2 Scope of Thesis 7

$\begin{array}{lll}\text { CHAPTER } 2 & 10\end{array}$

2.1 The Hamiltonian 10

$\begin{array}{ll}2.2 & \text { Transformation to Equilibrium } \\ \text { Coordinate Axes } & 12\end{array}$

2.3 Transformation to Fourier-
transformed Spin operators

2.4 Conditions for Stable Equilibrium $\quad 16$

2.5 Equations of Motion and Their Linearization 21

2.6 Solution of the Eigenvalue Problem 26

$\begin{array}{lll}\text { CHAPTER } 3 & \text { MAGNETOELASTIC EFFECTS OF HIGHER ORDER } & 33\end{array}$

3.1 The Theory of Callen and Callen 33

3.2 The Frozen Lattice Approximation 37

3.3 The Mobile Lattice Approximation 48

CHAPTER 4 ANALYSIS OF PARTICULAR SPIN STRUCTURES 53

4.1 The Planar Ferromagnet 53

4.2 The Nonplanar Ferromagnet 57

4.3 The Planar Spiral 59

4.4 The Conical Spiral 60 
$\underline{\text { Page }}$

APPENDIX A STABILITY CONDITION AT FINITE TEMPERATURES

62

APPENDIX B RANDOM PHASE APPROXIMATION AND LINEARIZATION OF THE EQUATIONS OF MOTION

APPENDIX C TRANSFORMATION OF SPIN OPERATOR EQUIVALENTS

68

REFERENCES

75 
ILIUSTRATIONS

$\underline{\text { Page }}$

FIGURE 1 FIRST BRILLOUIN ZONE FOR HCP STRUCTURE

29

vi 
TABLES

TABLE 1 MAGNETOSTRICTION CONSTANTS

Page

44

vii 


\section{CHAPTER 1}

\section{INTRODUCTION}

\subsection{Magnetic Interactions}

The magnetic moments on atoms in solids may interact with each other in a number of ways. However, the simplest model of magnetism in metals is the following: electrons in well localized magnetic $d$ or $f$ shells interact with one another via a Heisenberg exchange mechanism, while a distinct set of electrons in Bloch states accounts for the metallic properties. Hence, we distinguish between localized magnetic electrons which carry the major part of the bulk magnetic moment and the itinerant conduction electrons which contribute little magnetic moment. The low-lying excitations of spin systems coupled by exchange interactions are wave-like and are called spin waves. In a spin wave a small sinusoidally varying deviation of the moment from the completely ordered state travels through the lattice. The energy of a spin wave is quantized in units of energy called magnons and the excitation of a single magnon reduces the ordered moment of the system by one Bohr magneton. If the interactions between magnons are neglected then they have a well defined energy $E_{q}$ and momentum $\hbar \mathrm{q}$. Exchange forces determine the dispersion relation $\mathrm{E}_{\mathrm{g}}$ vs $\mathrm{q}$ primarily and hence 
a determination of the dispersion curve by neutron inelastic scattering will give information about the magnetic interactions.

In the past decade the heavy rare earth metals have stimulated a great deal of interest due to their rich variety of equilibrium magnetic configurations as revealed by neutron diffraction experiments. In all of the various moment arrangements, the moments of the ions lying in a given hexagonal layer are parallel. However, the direction of the moment changes from one layer to the next and this variation of the moment along the hexagonal axis can be described by a wave vector $\underline{k}_{0}$ whose direction is parallel to this axis. A complete description of these various moment arrangements can be found in a review article by cooper (1968b).

In order to discuss the differences in magnetic behaviour of the various members of the heavy rare earth group we can look upon each metal as consisting of a lattice of localized rare earth tripositive ions immersed in a sea of conduction electrons. Within each ion the intra-atomic Coulomb interaction produces states characterized by $\underline{L}$ and $\underline{S}$ where $\underline{I}$ is the total orbital angular momentum and $\underline{S}$ is the total spin angular momentum. When the spin-orbit interaction is added, only the total angular momentum $\underline{J}=\underline{L}+\underline{S}$ is conserved. Upon forming the metal each atom lost its $5 d 6 s^{2}$ electrons to the conduction bands leaving behind a highly localized moment at each lattice site due to the unfilled 4 f shells. 
In general this moment is given by Hund's rules so that the total angular momentum J can be treated as a good quantum number.

Since the radius of the $4 f$ shell is about $0.35 \AA$, which is small compared to the interatomic distances of about $3.5 \mathrm{~A}$, the overlap of the $4 \mathrm{f}$ wave functions from two neighbouring atoms is very small and direct interaction between the magnetic moments is negligible. The observed magnetism must then involve the conduction electrons which must be capable of sustaining correlations of the moments over several interatomic distances. While a conduction electron is near a particular lattice site the coupling with the localized $f$ electrons is like an intra-atomic exchange, being summed over all f electrons at that site

$$
\text { [1.1] } \quad \sum_{\mathrm{f}} \mathrm{K} \underline{\underline{s}}_{\mathrm{C}} \cdot \underline{\underline{s}}_{\mathrm{f}}=K \underline{\mathrm{s}}_{\mathrm{C}} \cdot \underline{\underline{s}}=K(g-1) \underline{\underline{s}}_{\mathrm{C}} \cdot \underline{\mathrm{J}} \text {. }
$$

Here $\mathrm{K}$ is the intra-atomic exchange constant between the $4 \mathrm{f}$ and $6 \mathrm{~s}$ electrons; $\underline{s}_{c}$ is the conduction electron spin; $\underline{S}$ is the total ionic spin; and $(g-1) \mathrm{J}$ is the projection of $\underline{\mathrm{S}}$ onto the total angular momentum of the ion. In second order perturbation theory this interaction causes an effective interaction between ionic moments. The localized 4 f moments produce around them a spin polarization of the conduction system which in turn interacts with another localized moment and gives rise to an indirect exchange interaction between the two moments. It can be shown that the indirect exchange 
may be written as a Heisenberg exchange interaction (Ruderman and Kittel 1954, Kasuya 1956, Yosida 1957).

$[1.2]$

$$
\mathcal{F}_{\text {ex }}=-\sum_{i<j} J_{i j}\left(R_{i j}\right) \underline{S}_{i} \cdot \underline{S}_{j} \text {. }
$$

If the conduction electrons are treated using the free electron model, then $J(R)$ has the following form:

$$
J(R)=\frac{3 z \pi K^{2}}{2 E_{F}}(g-1)^{2} F\left(2 k_{F} R\right)
$$

$$
F(Y)=(Y \cos Y-\sin Y) / Y^{4}
$$

where $\mathrm{K}$ is the $\mathrm{s} f \mathrm{f}$ exchange interaction; $\mathrm{z}$ is the number of conduction electrons; and $E_{F}$ is the Fermi energy. In order to consider the dependence of energy on $q$ it is convenient to define the fourier transform of $J(R)$

$$
J(q)=\sum_{j} J\left(R_{i j}\right) e^{i q \cdot\left(\underline{R}_{i}-R_{j}\right)} .
$$

For the free electron model, this has the form:

$$
\text { [1.5a] } J(q)=\frac{3 z K^{2}}{16 E_{F}}(g-1)^{2}\left\{1+\frac{1-x^{2}}{2 x} \log \frac{1+x}{1-x}\right\}
$$

where,

$[1.5 b]$

$$
\mathrm{x}=\mathrm{q} / 2 \mathrm{k}_{\mathrm{F}}
$$

Although $J(R)$ is long ranged and oscillatory, the maximum of 
$J(q)$ falls at $q=0$. However, Yosida and Watabe (1962) have used a nearly free electron model for the conduction band to show that $J(\underline{q})$ can have its maximum at a non-zero wave vector $k_{0}$ along the c-axis if the periodicity of the hexagonal close-packed lattice is considered. It is the exchange interactions which determine the periodicity of the equilibrium magnetic moment arrangement.

The exchange interaction is however not sufficient to describe the magnetism in the rare earths. Since the orbital angular momentum $\underline{L}$ of each ion is not quenched, the distribution of $4 \mathrm{f}$ electrons is not spherical but is expanded in a plane normal to J. This results in an anisotropic crystalline field (Elliott 1961, Miwa and Yosida 1961) which is large and cannot be neglected. If the neighbouring ions are treated as point charges, the crystal field can be expanded in terms of spherical harmonics and will have the same symmetry as the ionic lattice. For the heavy rare earths this structure is hexagonal close-packed (hcp) with the c/a ratio between 1.57 and 1.59 . Since the lowest $J$ multiplet is usually more than about $0.1 \mathrm{eV}$ below the next excited multiplet and since the splittings of these multiplets by the crystal field are of the order of $0.01 \mathrm{eV}$, we can consider the ions as being confined to the lowest $J$ multiplet. The matrix elements of the crystal field within this J manifold are then easily evaluated using the method of operator equivalents (see for example Hutchings 1964). 
Another important contribution to the anisotropy energy is magnetostriction. The crystal structure in ferromagnets is more or less distorted compared to the paramagnetic state and the distortion depends on the direction of the spontaneous magnetization. The deformation is expressed by the change of linear dimension measured along a certain direction and is dependent on both the direction of magnetization and the direction of observation. In order to express the dependence of the anisotropy energy on the strain, we expand the energy in a Taylor's series in the strains. The terms linear in the strains refer to the deformed lattice which has lower symmetry than the unstrained crystal. This coupling between the strains and the direction of magnetization is called magnetoelastic coupling. These terms correspond to the anisotropic crystal field of the distorted lattice and can be expanded in terms of a complete set of spherical harmonics having the distorted lattice symmetry (Lindgard 1971). Thus they can be handled using the operator equivalent method also.

Both the crystal field anisotropy and magnetostriction compete with exchange interactions to determine the equilibrium magnetic moment configuration. Exchange favours a spiral spin arrangement while axial anisotropy terms determine whether the arrangement is planar or conical. Even when exchange favours a spiral spin arrangement, planar anisotropy terms and magnetostriction effects can overcome this 
tendency and give ferromagnetism.

1.2 Scope of Thesis

A general theory of spin waves for the helical spin structures found in the heavy rare earth metals was first developed by Cooper, Elliott, Nettel and Suhl (1962). Starting from a Hamiltonian consisting of Heisenberg exchange coupling and single-ion crystal field terms they obtained expressions for the spin wave energies for various types of magnetic ordering. These expressions have often been used to analyze the results of magnetic resonance and inelastic neutron scattering experiments.

Recent experimental work has shown the need for a theory extending the work of cooper et. al. in a number of ways. First, there is the trivial point that the hcp crystal structure of heavy rare earth metals has two atoms per unit cell, whereas Cooper et. al. assumed a Bravais lattice for simplicity. As a result they did not obtain expressions for the higher spin-wave mode. Secondly, it is desirable to treat the crystal field terms more carefully than was done by Cooper et. al. whose expressions are correct only to the highest power of $\mathrm{S}$, the total angular momentum. Careful manipulation of the spin commutation relations, however, yields expressions with desirable symmetry properties. Thirdly, it has been recognized in recent years (Turov and Shavrov 1965, Cooper 1968a) that the magnetoelastic coupling between the magnetic spins and the lattice has an important 
influence on the spin-wave energies. In fact, the experimental work of Rhyne and Clark (1967) and of Nielsen et. al. (1970) shows the need to consider magnetoelastic contributions of higher order than those discussed by cooper (1968a). Finally, the temperature dependence of the spin-wave energies measured by magnetic resonance and neutron scattering experiments requires for its description a spin-wave theory incorporating in some approximate manner the effect of spin-wave interactions. While some of these extensions appear in recent theoretical papers concerned with terbium and dysprosium (Brooks, Goodings and Ralph 1968, Cooper 1968, Brooks 1970, Lindgard 1971) a theory having all these features has not so far been published.

In the present work a spin-wave theory is presented for the general conical spiral structure which includes the improvements mentioned in the preceeding paragraph. This general magnetic structure contains the planar ferromagnet, the planar spiral and the nonplanar ferromagnet as special cases. Excluded from the theory are the distorted spiral and fan structures which result when a helical spin structure is placed in a magnetic field perpendicular to the hexagonal axis (Enz 1960), Cooper and Elliott 1963, Nagamiya 1967). Also excluded are the oscillatory z-component or sinusoidal spin arrangement found in erbium between $53.5^{\circ} \mathrm{K}$ and $85^{\circ} \mathrm{K}$ and in thulium between $40^{\circ} \mathrm{K}$ and $56^{\circ} \mathrm{K}$, and the type of antiphase domain structure found in thulium below about $40^{\circ} \mathrm{K}$. 
In Chapter 2, the derivation of the spin wave energies is outlined. The equations of motion for the spin operators are linearized by means of approximations equivalent to the random phase approximation (Englert 1960, Brooks 1970), the effect of higher order terms being expressed in an approximate way through powers of the reduced magnetization. In Chapter 3 higher order magnetoelastic terms corresponding to spherical harmonics with $l=4$ are discussed in detail and their contributions to the spin-wave energies are written down. Finally, in Chapter 4 explicit expressions for the spin-wave energies for the four magnetic structures under consideration are given. 
CHAPTER 2

SPIN WAVE THEORY

Because the calculation of the spin-wave energies is rather lengthy we shall not quote any intermediate results. However, it should be clear from what follows how the final results were obtained.

\subsection{The Hamiltonian}

The magnetic properties of the rare earth metals of hcp structure are described by the Hamiltonian (Cooper, Elliott, Nettel and Suhl, 1962; Cooper 1968a).

$$
\begin{aligned}
& \mathcal{H}=-\sum_{i<j} J_{i j} \underline{S}_{i} \cdot \underline{S}_{j}-\sum_{i<j} K_{i j} s_{i}^{\zeta} S_{j}^{\zeta}+g \mu_{B} \underline{H} \cdot\left(\sum_{i} \underline{S}_{i}\right) \\
& \left.+\widetilde{\mathrm{B}}_{2}^{0} \sum_{i} \widetilde{\mathrm{O}}_{20}+\widetilde{\mathrm{B}}_{4}^{0} \sum_{i} \widetilde{\mathrm{O}}_{40}+\widetilde{\mathrm{B}}_{6}^{0} \sum_{i} \widetilde{\mathrm{O}}_{60}+\widetilde{\mathrm{B}}_{6}^{6} \sum_{i} \widetilde{\mathrm{O}}_{66}+\widetilde{\mathrm{O}}_{6-6}\right) \\
& -\widetilde{B}^{\gamma}\left[\bar{\varepsilon}_{1}^{\gamma} \sum_{i} \frac{1}{2}\left(S_{i}^{\xi 2}-s_{i}^{n 2}\right)+\bar{\varepsilon}_{2}^{\gamma} \sum_{i} \frac{1}{2}\left(s_{i}^{\xi} s_{i}^{n}+s_{i}^{n} s_{i}^{\xi}\right)\right] \\
& {[2.1]} \\
& -\widetilde{B}^{\varepsilon}\left[\bar{\varepsilon}_{1}^{\varepsilon} \sum_{i} \frac{1}{2}\left(S_{i}^{n} S_{i}^{\zeta}+S_{i}^{\zeta} S_{i}^{n}\right)+\bar{\varepsilon}_{2}^{\varepsilon} \sum_{i} \frac{1}{2}\left(s_{i}^{\xi} s_{i}^{\zeta}+S_{i}^{\zeta} s_{i}^{\xi}\right)\right] .
\end{aligned}
$$

The first term is the usual Heisenberg exchange coupling predicted by the theory of indirect exchange (see the review article by Kittel (1968)) while the term in $\mathrm{K}_{i j}$ is included to take account of any anisotropic exchange which may be present. The terms in $\widetilde{\mathrm{B}}_{2}^{0}, \widetilde{\mathrm{B}}_{4}^{0}$ and $\widetilde{B}_{6}^{0}$ describe a crystal field with axial symmetry while the term in $\widetilde{B}_{6}^{6}$ describes the hexagonal anisotropy. To avoid confusion 
with the exchange constants $\underline{S}_{i}$ is used for the total angular momentum of atom $i$, including orbital and spin contributions. The $\xi \eta \zeta$ coordinate system has the $\zeta$-axis along the hexagonal crystal axis and the $\xi$-axis directed toward a nearest neighbor atom in the basal plane. The $\widetilde{O}_{\ell m}$ are the spin operator equivalents tabulated by Buckmaster (1962) and Smith and Thornley (1966) and denoted "Racah operator equivalents" by Birgeneau (1967) who has given numerical tables of their matrix elements ${ }^{\dagger}$.

$t_{\text {The }} \widetilde{O}_{\ell m}$ are related to the operator equivalents of stevens (1952) by constant factors chosen so that they transform under rotations in exactly the same way as do the spherical harmonics. As a result they satisfy the commutation relations (2.22) and (2.23) for tensor operators (Edmonds $1957, \mathrm{p} .71$ ) which greatly simplifies the algebra involved in the calculation. Note, however, that they do not contain the factor $(2 \ell+1 / 4 \pi)^{1 / 2}$ which occurs in the usual definition of the spherical harmonics $Y_{\ell m}(\theta, \phi)$. The operator equivalents $o_{\ell}^{m}$ used by Brooks $(1969,1970)$, by Brooks and Goodings (1969) and by Brooks, Goodings and Ralph (1968) are exactly the same as the $\vec{\sigma}_{\ell}^{m}$ of orbach (1961). The operator equivalents and crystal field parameters of the present paper are related to those in the earlier papers by

$$
\begin{array}{rlrl}
\widetilde{\mathrm{o}}_{20} & =\frac{1}{2} \mathrm{O}_{2}^{0}, & \widetilde{\mathrm{B}}_{2}^{0} & =2 \mathrm{~B}_{2}^{0} \\
\widetilde{\mathrm{o}}_{40} & =\frac{1}{8} \mathrm{O}_{4}^{0}, & \widetilde{\mathrm{B}}_{4}^{0} & =8 \mathrm{~B}_{4}^{0} \\
\widetilde{\mathrm{o}}_{60} & =\frac{1}{16} \mathrm{o}_{6^{\prime}}^{0}, \widetilde{\mathrm{B}}_{6}^{0} & =16 \mathrm{~B}_{6}^{0} \\
\widetilde{\mathrm{o}}_{66}+\widetilde{\mathrm{o}}_{6-6} & =\left[(231)^{\frac{1}{2}} / 16\right] \mathrm{O}_{6}^{6} & \widetilde{\mathrm{B}}_{6}^{6} & =\left[16 /(231)^{\frac{1}{2}}\right]_{\mathrm{B}}^{6}
\end{array}
$$


The last two lines of [2.1] are magnetoelastic terms whose importance with regard to the spin wave energies of the rare earth metals and other crystals showing large magnetostrictive effects was first recognized by Turov and Shavrov (1965). They are written here in the form appropriate to the hcp lattice derived by Callen and Callen (1965) and discussed by Cooper (1968a). $\widetilde{\mathrm{B}}^{\gamma}$ and $\widetilde{B} \varepsilon$ are phenomenological magnetoelastic coupling constants and $\varepsilon_{1}^{\gamma}, \varepsilon_{2}^{\gamma}, \varepsilon_{1}^{\varepsilon}$ and $\varepsilon_{2}^{\varepsilon}$ are symmetrized strains defined in chapter 3 . Furthermore we have assumed that the strains are "frozen" at their equilibrium values, an approximation referred to by Turov and Shavrov and Cooper as the frozen lattice approximation. A full discussion of magnetostrictive terms in the Hamiltonian, including the applicability of the frozen lattice approximation, is given in Chapter 3. All possible single-ion magnetoelastic terms linear in the strains and up to fourth order in $s_{i}^{\xi}, s_{i}^{n}$ and $s_{i}^{\zeta}$ and all possible two-ion magnetoelastic terms linear in the strains and up to second order in the spin operators are considered in detail. The relationship between the equilibrium strains $\bar{\varepsilon}_{1}^{\gamma}, \bar{\varepsilon}_{2}^{\gamma}, \bar{\varepsilon}_{1}^{\varepsilon}$ and $\bar{\varepsilon}_{2}^{\varepsilon}$ and the saturation magnetostriction constants is also given in

Chapter 3. In this section of the paper, however, we shall include only the lowest-order single-ion magnetoelastic terms which make up the last two lines of [2.1].

2.2 Transformation to Equilibrium Coordinate Axes

A spin wave theory is constructed by considering the deviations of each spin from its equilibrium direction. Therefore 
we transform to local coordinate axes at each site (Yosida and Miwa 1961, Kaplan 1961, Cooper et. al. 1962) chosen so that the $z_{i}$-axis lies along the equilibrium direction of $\underline{S}_{i}$ and the $Y_{i}$-axis lies in the basal plane. The transformation is,

$$
s_{i}^{\xi}=s_{i}^{x} \cos \theta \cos \phi_{i}-s_{i}^{y} \sin \phi_{i}+s_{i}^{z} \sin \theta \cos \phi_{i}
$$

$$
s_{i}^{n}=s_{i}^{x} \cos \theta \sin \phi_{i}+s_{i}^{y} \cos \phi_{i}+s_{i}^{z} \sin \theta \sin \phi_{i}
$$

$$
s_{i}^{\zeta}=-s_{i}^{x} \sin \theta+s_{i}^{z} \cos \theta
$$

with $\theta$ the cone angle and $\phi_{i}=\underline{k}_{0} \cdot \underline{R}_{i}+\phi, \underline{k}_{0}$ being the wave vector of the spiral. Because of the experimental fact that the spins in each plane perpendicular to the hexagonal axis are aligned, $\underline{k}_{0}$ is assumed to be in the direction of the $\zeta$-axis.

The transformation [2.2] is equivalent to a rotation of the $\xi n \zeta$ coordinate system through the Euler angles $\alpha_{i}=\phi_{i}, \beta_{i}=\theta$, $\gamma_{i}=0$. Under these rotations the $\widetilde{o}_{\ell m}$ transform according to [2.3]

$$
\widetilde{o}_{\ell m}\left(s_{i}^{\xi}, s_{i}^{\eta}, s_{i}^{\zeta}\right)=\sum_{m^{\prime}} D_{m}^{(\ell)}\left(\phi_{i} \theta 0\right) \widetilde{o}_{\ell m},\left(s_{i}^{x}, s_{i}^{Y}, s_{i}^{z}\right)
$$

where the $D_{m}^{(\ell)}\left(\alpha_{i} \beta_{i} \gamma_{i}\right)$ are the standard rotation matrices (we follow the notation and conventions of Edmonds (1957)). The result of applying this transformation to the Hamiltonian gives rather lengthy expressions, particularly for the term in $\widetilde{\mathrm{B}}_{6}^{6}$ for which the rotation matrices do not assume a simple form. These will be given in Appendix $C$. 
The approximate ground state of the system at $T=0$, denoted $|0\rangle$, is chosen to have $m_{s}=-s$ for each spin, ie.

$[2.4 \mathrm{a}]$

$$
\begin{aligned}
& s_{i}^{z}|0>=-s| 0> \\
& s_{i}^{-} \mid 0>=0 .
\end{aligned}
$$

$[2.4 \mathrm{~b}]$

In what follows we shall make use of an expansion of $s_{i}^{z}$ due to Wortis (1963)

$$
s_{i}^{z}=-s+(2 S)^{-1} s_{i}^{+} s_{i}^{-}+(2 S)^{-2}(2 s-1)^{-1} s_{i}^{+} s_{i}^{+} s_{i}^{-} s_{i}^{-}+\cdots
$$

2.3 Transformation to Fourier-Transformed Spin Operators

Since the hexagonal crystal structure can be decomposed into two interpenetrating sublattices, it is convenient to define two sets of Fourier-transformed spin operators,

$$
\mathrm{s}_{\underline{\underline{q}}}^{ \pm}=\mathrm{N}^{-\frac{1}{2}} \sum_{i} \mathrm{~s}_{i}^{ \pm} \exp \left( \pm i \underline{\left.\underline{q} \cdot \underline{r}_{i}\right)}\right.
$$

$$
\begin{aligned}
& s_{\underline{q}}^{z}=N^{-1} \sum_{i} s_{i}^{z} \exp \left(-i q \cdot \underline{r}_{i}\right) \\
& T_{\underline{q}}^{ \pm}=N^{-\frac{1}{2}} \sum_{j} s_{j}^{ \pm} \exp \left( \pm i q \cdot \underline{r}_{j}\right)
\end{aligned}
$$

$$
T_{\underline{q}}^{z}=N^{-1} \sum_{j} s_{j}^{z} \exp \left(-i \underline{q} \cdot \underline{r}_{j}\right)
$$

where $s_{i}^{ \pm}=s_{i}^{x} \pm i s_{i}^{Y}$ and the sum over $i$ runs over one sublattice, the sum over $j$ over the other sublattice. $N$ is the number of unit cells in the crystal. From the usual commutation rules of the spin 
operators one finds

$[2.7 \mathrm{a}]$

$$
\begin{aligned}
& {\left[s_{\underline{q}^{\prime}}^{+}, S_{\underline{q}^{\prime}}^{-}\right]=2 S_{\underline{q}^{\prime}-q}^{z}} \\
& {\left[S_{\underline{q}^{\prime}}^{z} s_{\underline{q}^{\prime}}^{ \pm}\right]= \pm N^{-1} S_{\underline{q}^{\prime} \mp q}^{ \pm}}
\end{aligned}
$$

$[2.7 \mathrm{~b}]$

with similar relations for the operators $\mathrm{T}_{\mathrm{q}^{\prime}}^{+} \mathrm{T}_{\mathrm{q}}^{-}$and $\mathrm{T}_{\mathrm{q}}^{\mathrm{z}}$. Since the $\mathrm{S}_{\mathrm{q}}$-operators and $\mathrm{T}_{\mathrm{q}}$-operators involve spins on different sublattices they commute with each other.

By means of the transformation inverse to [2.6] the Hamiltonian is expressed in terms of these Fourier transformed operators and the functions

$$
\begin{aligned}
& \text { [2.8a] } \\
& J(\underline{q})=\sum_{i}, J_{i i}, \exp \left\{i \underline{q} \cdot\left(\underline{r}_{i}-\underline{r}_{i}{ }^{\prime}\right)\right\} \\
& \text { [2.8b] } \\
& J^{\prime}(\underline{q})=\sum_{j} J_{i j} \exp \left\{i \underline{q} \cdot\left(\underline{r}_{i}-\underline{r}_{j}\right)\right\} \\
& \text { [2.8c] } \\
& K(\underline{q})=\sum_{i}, K_{i i}, \exp \left\{i \underline{q} \cdot\left(\underline{r}_{i}-\underline{r}_{i}{ }^{\prime}\right)\right\} \\
& \text { [2.8d] } \\
& K^{\prime}(\underline{q})=\sum_{j} K_{i j} \exp \left\{i \underline{q} \cdot\left(\underline{r}_{i}-\underline{r}_{j}\right)\right\}
\end{aligned}
$$

In these definitions $i$ ' runs over the same sublattice as $i$ whereas $j$ runs over the other sublattice. Here also we shall not give the expressions obtained.

We note that the Fourier transform of the Wortis expansion $[2.5]$ is,

$$
s_{\underline{q}}^{z}=-s \delta_{\underline{q}, 0}+(2 S N)^{-1} \sum_{\underline{q}^{\prime}} S_{\underline{q}^{\prime}-\underline{q}^{+} \underline{q}^{\prime}}^{s^{-}+\ldots}
$$

with a similar expression for $\mathrm{T}_{\underline{\mathrm{q}}}^{\mathrm{z}}$. Since the z-axis at each ion 
makes an angle $\theta$ with respect to the hexagonal axis, the component of the magnetization parallel to this axis is,

$[2.10] \quad M_{\|}(T)=-2 g \mu_{B} \cos \theta<\underset{i}{\cos s_{i}^{z}}>/ V$

where $\mathrm{V}$ is the volume, < denotes a thermal average and the factor of 2 compensates for the sum over i running over only one sublattice. The reduced component of magnetization is therefore

$$
\begin{aligned}
& m_{\|}(T) \equiv \frac{M \|(T)}{M_{\|}(0)}=-(N S)^{-1}\left\langle\underset{i}{\left\langle S_{i}^{z}\right\rangle}=-S^{-1}\left\langle S_{0}^{z}\right\rangle .\right. \\
& =-S^{-1}\left\langle T_{0}^{z}\right\rangle
\end{aligned}
$$

Now if $\left\langle\mathrm{S}_{0}^{\mathrm{z}}\right\rangle$ is approximated by the first two terms of [2.9] then one obtains the relation

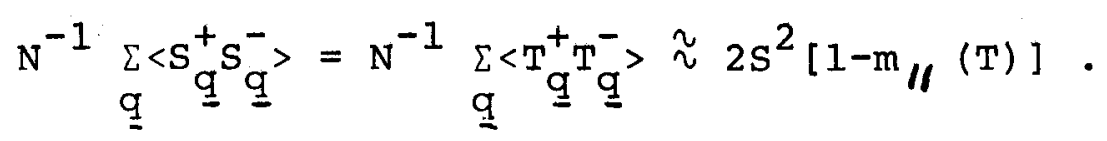

The same relation may be shown to hold for the case of a ferromagnet with the spins lying in the plane $\left(\theta=\frac{\pi}{2}\right)$, the total reduced magnetization $m(T)$ replacing $m /(T)$ in this case (Brooks 1970). This relation provides a simple means of introducing some temperature dependence into the theory.

2.4 Conditions for Stable Equilibrium

By definition the equilibrium axis at each site must be such that the free energy is a minimum with respect to variation of $\theta$ and $\phi_{i}$. Since the present work includes some interaction effects, 
leading to temperature dependent spin-wave energies, it is necessary to include the entropy term in the free energy. In Appendix A it is argued that expressions consistent within the present theory are obtained by differentiating only those terms in $\langle\boldsymbol{K}\rangle$ which are explicit functions of $\theta$ and $\phi_{i}$, excluding from the differentiation factors which depend implicitly on $\theta$ and $\phi_{i}$ through thermal averages.

Let us consider the contribution to the thermal average $\langle\boldsymbol{X}\rangle$ from two-ion terms. We first substitute the first two terms of the expansion [2.9] for $\mathrm{S}_{\underline{q}}^{\mathrm{z}}$ and $\mathrm{T}_{\mathrm{q}}^{\mathrm{z}}$ wherever they occur, discarding all terms beyond those quadratic in the operators $\mathrm{s}_{\mathrm{q}}^{+}$, $\mathrm{S}_{\mathrm{q}^{\prime}}^{-} \mathrm{T}_{\underline{q}}^{+}$and $\mathrm{T}_{\underline{q}}^{-}$. We then call on the random phase approximation to discard all terms $\left\langle\mathrm{S}_{\underline{q}_{1}}^{+} \mathrm{S}_{\underline{q}_{2}}^{-}\right\rangle$and $\left\langle\mathrm{T}_{\mathrm{q}_{1}}^{+}{ }_{\mathrm{T}_{\mathrm{q}}^{-}}^{-}>\right.$except those with $\underline{\mathrm{q}}_{1}=\mathrm{q}_{2}$. In fact the only higher orders terms we retain are those involving $\mathrm{N}^{-1} \Sigma\left\langle\mathrm{S}_{\mathrm{q}}^{+} \mathrm{S}^{-}\right\rangle$and $\mathrm{N}^{-1} \Sigma\left\langle\mathrm{T}_{\mathrm{q}}^{+} \mathrm{T}_{\mathrm{q}}^{-}\right\rangle$since for these we can use the relation

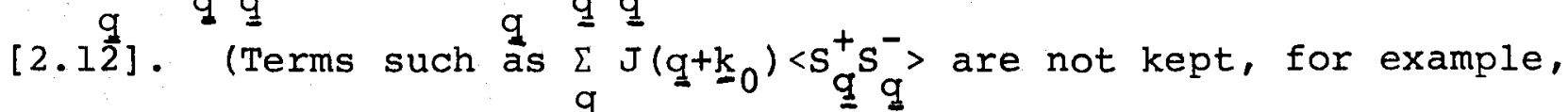
because they cannot readily be expressed in terms of the reduced magnetization.)

The contributions to $\langle\boldsymbol{X}\rangle$ from the crystal field and singleion magnetoelastic terms are handled similarly, but as in the papers by Kittel and Van Vleck (1960) and Callen and Callen (1966), only terms with axial symmetry about the equilibrium direction of the spins are retained. From [2.3],

$[2.13 a]$

$$
<\widetilde{O}_{\ell m}\left(s_{i}^{\xi}, s_{i}^{n}, s_{i}^{\zeta}\right)>=D_{0 m}^{(\ell)}\left(\phi_{i}, \theta, 0\right)<\widetilde{O}_{\ell 0}\left(s_{i}^{X}, s_{i}^{Y}, s_{i}^{z}\right)>
$$

$$
\left.=[4 \pi /(2 \ell+1)]^{\frac{1}{2}} Y_{\ell m}\left(\theta, \phi_{i}\right)<\widetilde{o}_{\ell 0}\left(S_{i}^{X}, S_{i}^{Y}, s_{i}^{z}\right)\right\rangle
$$


where we are using the convention of Edmonds (1957). The thermal average on the right hand side of this equation is calculated in Appendix B, the temperature dependence being expressed through powers of the reduced magnetization. We find,

$$
<\widetilde{O}_{\ell 0}\left(s_{i}^{x}, s_{i}^{y}, s_{i}^{z}\right)>\approx s s((\ell-I) / 2) \mathrm{m}^{\ell(\ell+1) / 2}
$$

where $S(n)$ is defined below in $[2.16]$.

Making these approximations and carrying out the differentiation with respect to $\theta$ we find the following condition for stable equilibrium,

$$
\begin{aligned}
& S \sin \theta \cos \theta\left[J(0)+K(0)+J^{\prime}(0)+K^{\prime}(0)-J\left(\underline{k}_{0}\right)-J^{\prime}\left(\underline{k}_{0}\right)\right] m^{2} \\
& -\widetilde{B}_{2}^{0} S(1 / 2) P_{2}^{1} m^{3}-\widetilde{B}_{4}^{0} S(3 / 2) P_{4}^{1} m^{10}-\widetilde{B}_{6}^{0} S(5 / 2) P_{6}^{1} m^{21} \\
& +6 \mathrm{~B}_{6}^{6} \sin ^{5} \theta \cos \theta \cos 6 \phi \mathrm{s}(5 / 2) \mathrm{m}^{21} \delta_{\mathrm{k}_{0}, 0} \\
& +g \mu_{B} H_{\zeta} \sin \theta m-g \mu_{B}\left(H_{\xi} \cos \phi+H_{n} \sin \phi\right) \cos \theta m \delta_{\underline{k}_{0}, 0} \\
& -\widetilde{B}^{\gamma} \sin \theta \cos \theta\left(\bar{\varepsilon}_{I}^{\gamma} \cos 2 \phi+\bar{\varepsilon}_{2}^{\gamma} \sin 2 \phi\right) \mathrm{s}(1 / 2) \mathrm{m}^{3} \delta_{\mathrm{k}_{0}, 0} \\
& {[2.15]} \\
& -\widetilde{B}^{\varepsilon} \cos 2 \theta\left(\bar{\varepsilon}_{1}^{\varepsilon} \sin \phi+\bar{\varepsilon}_{2}^{\varepsilon} \cos \phi\right) \mathrm{s}(1 / 2) \mathrm{m}^{3} \delta_{\underline{k}_{0}, 0}=0
\end{aligned}
$$

where,

$$
\begin{aligned}
S(n) & =\left(S-\frac{1}{2}\right)(S-1) \ldots(S-n) \\
B_{6}^{6} & =(231)^{\frac{1}{2}} \widetilde{B}_{6}^{6} / 16
\end{aligned}
$$

$\mathrm{H}_{\xi}, \mathrm{H}_{\eta}$ and $\mathrm{H}_{\zeta}$ are the components of the applied field in the original 
coordinate system referred to crystalline axes. In [2.15] $\mathrm{m}$ stands for $m \|(T)$ when $\theta \neq \pi / 2$ and for $m(T)$ when $\theta=\pi / 2$, for the reason $g i v e n$ in section 2.3, and $\mathrm{P}_{\ell}^{\mathrm{m}}$ stands for the associated Legendre function $\mathrm{P}_{\ell}^{\mathrm{m}}(\cos \theta)$. The factor $\delta_{\underline{\mathrm{k}}_{0}, 0}$ has been introduced into the term in $B_{6}^{6}$ in order to exclude the hexagonal anistropy from spiral structures, for the reason given in the next subsection. The magnetoelastic terms must also be excluded in considering spiral structures because homogeneous strains cannot be defined. The same factor occurs in the magnetic field term involving $\mathrm{H}_{\xi}$ and $\mathrm{H}_{\eta}$ as a result of averaging over all sites.

We shall find it useful to re-express $\mathrm{P}_{2}^{1}, \mathrm{P}_{4}^{1}$ and $\mathrm{P}_{6}^{1}$ by means of the recurrence relation

$$
2 m \cos \theta P_{\ell}^{m}=\sin \theta\left[P_{\ell}^{m+1}+(\ell+m)(\ell-m+1) P_{\ell}^{m-1}\right] .
$$

After multiplying [2.15] by $\cot \theta$ and rearranging the terms we obtain the condition for stable equilibrium in the form,

$$
\begin{aligned}
& 3 \widetilde{\mathrm{B}}_{2}^{0} \mathrm{~S}(1 / 2) \mathrm{P}_{2}^{0} \mathrm{~m}^{3}+1 \widetilde{\mathrm{B}}_{4}^{0} \mathrm{~s}(3 / 2) \mathrm{P}_{4}^{0} \mathrm{~m}^{10}+2 \widetilde{\mathrm{B}}_{6}^{0} \mathrm{~s}(5 / 2) \mathrm{P}_{6}^{0} \mathrm{~m}^{21} \\
&+ \frac{1}{2}\left[\widetilde{\mathrm{B}}_{2}^{0} \mathrm{~s}(1 / 2) \mathrm{P}_{2}^{2} \mathrm{~m}^{3}+\widetilde{\mathrm{B}}_{4}^{0} \mathrm{~s}(3 / 2) \mathrm{P}_{4}^{2} \mathrm{~m}^{10}+\widetilde{\mathrm{B}}_{6}^{0} \mathrm{~s}(5 / 2) \mathrm{P}_{6}^{2} \mathrm{~m}^{21}\right] \\
&=\mathrm{S} \cos ^{2} \theta\left[\mathrm{J}(0)+\mathrm{K}(0)+\mathrm{J}^{\prime}(0)+\mathrm{K}^{\prime}(0)-\mathrm{J}\left(\underline{\mathrm{k}}_{0}\right)-\mathrm{J}^{\prime}\left(\underline{\mathrm{k}}_{0}\right)\right] \mathrm{m}^{2} \\
&+6 \mathrm{~B}_{6}^{6} \sin ^{4} \theta \cos ^{2} \theta \cos 6 \phi \mathrm{s}(5 / 2) \mathrm{m}^{21} \delta_{\underline{k}_{0}, 0} \\
&+\mathrm{g} \mu_{\mathrm{B}} \mathrm{H}_{\zeta} \cos \theta \mathrm{m}-\mathrm{g} \mu_{\mathrm{B}}\left(\mathrm{H}_{\xi} \cos \phi+\mathrm{H}_{n} \sin \phi\right) \cos \theta \cot \theta \mathrm{m} \delta_{\mathrm{k}_{0}, 0} \\
&-\widetilde{\mathrm{B}}^{\gamma} \cos ^{2} \theta\left(\bar{\varepsilon}_{1}^{\gamma} \cos 2 \phi+\bar{\varepsilon}_{2}^{\gamma} \sin 2 \phi\right) \mathrm{s}(1 / 2) \mathrm{m}^{3} \delta_{\underline{\mathrm{k}}_{0}, 0} \\
& {[2.19] \quad-\widetilde{\mathrm{B}}^{\varepsilon} \cos 2 \theta \cot \theta\left(\bar{\varepsilon}_{1}^{\varepsilon} \sin \phi+\bar{\varepsilon}_{2}^{\varepsilon} \cos \phi\right) \mathrm{s}(1 / 2) \mathrm{m}^{3} \delta_{\underline{\mathrm{k}}_{0}, 0} }
\end{aligned}
$$


Since $m$ varies with temperature this condition may be regarded as determining $\theta$ as a function of temperature.

It should be mentioned at this point that the contribution to this condition from higher order magnetoelastic terms is set out in $[3.21]$.

The azimuthal angles $\phi_{i}$ must also be such as to give a stable equilibrium. Instead of defining $\phi_{i}=\underline{k}_{0} \cdot \underline{\underline{x}}_{i}+\phi$ as in [2.2] we can let each $\phi_{i}$ be an independent parameter whose value is to be determined so as to minimize the free energy. The resulting stability condition at each site represents a balance of the forces due to exchange (tending to produce the spiral), hexagonal anisotropy, magnetostriction and applied field. As it also involves the $\phi_{j}$ at neighboring sites we must solve a set of coupled equations. Cooper et. al. (1962) treated such a set of equations by an iterative procedure starting from the zeroth order solution $\phi_{i}=\underline{k}_{0} \cdot \underline{r}_{i}$, where $\underline{k}_{0}$ is defined to be the wave vector at which $J(\underline{q})$ is a maximum. Although they encountered considerable difficulties (see section 3.4 of their paper) they succeeded in deriving an expression for the departure of $\phi_{i}$ from $\underline{k}_{0} \cdot \underline{r}_{i}$ which is first order in $\mathrm{H}_{\perp}$ or $\mathrm{B}_{6}^{6}$. We shall not pursue this further but will simply assume that the structure is stable with respect to small variations in $\phi_{i}$ at each site. The value of $\underline{k}_{0}$ in spiral structures will be taken from experiment.

In the simple case of a ferromagnetic structure $\left(\underline{k}_{0}=0\right)$, the angle $\phi$ of the transformation [2.2] is the same at each site. 
Then the differentiation with respect to $\phi$ gives,

$$
\text { g } \mu_{B}\left(H_{\xi} \sin \phi-H_{n} \cos \phi\right) \sin \theta m-6 B_{6}^{6} \sin ^{6} \theta \sin 6 \phi s(5 / 2) m^{21}=0
$$

$[2.20]$

where $\mathrm{H}_{\xi}$ and $\mathrm{H}_{\eta}$ may have arbitrary values. This expresses the competing effects of the hexagonal anisotropy and of the components of $\mathrm{H}$ in the hexagonal plane in determining the equilibrium value of $\phi$. The magnetoelastic terms of [2.1], evaluated in the frozen lattice approximation (section 3.2), do not contribute to this equation, but there is a contribution from higher order terms (see equation [3.22]).

It should be added that equations [2.15] and [2.19] in the limit $\mathrm{T}=0$ may be obtained directly by keeping only the first term in the Wortis expansion [2.9]. In this limit they are essentially the same as the condition obtained by Cooper et. al. (1962) when allowance is made for the approximations of their paper. Furthermore equations [2.15] and [2.20] are exactly the conditions required to ensure that the terms in the transformed Hamiltonian linear in $\mathrm{S}_{\mathrm{q}^{\prime}}^{+} \mathrm{S}_{\mathrm{q}^{\prime}}^{-} \mathrm{T}_{\mathrm{q}}^{+}$and $\mathrm{T}_{\underline{\mathrm{q}}}^{-}$have vanishing coefficients, a property which is essential in constructing any spin-wave theory.

\subsection{Equations of Motion and their Linearization}

Equations of motion for the Fourier-transformed spin operators were first studied by Englert (1960) and by Ginzburg and Fain (1961) who linearized the equations by means of a simple random phase approximation. The advantages of this method compared 
with other methods for obtaining temperature-dependent spin-wave energies have been discussed by Brooks (1970) in a recent article concerned with spin-waves in terbium and dysprosium. We shall use this approach to obtain the spin-wave energies from the equations of motion,
$[2.21 a]$
it $\dot{\mathrm{s}}_{\mathrm{q}}^{-}=\left[\mathrm{s}_{\mathrm{q}^{-}}, \mathcal{H}\right]$
[2.21b]
ih $\dot{\mathrm{s}}_{-\mathrm{q}}^{+}=\left[\mathrm{s}_{-\mathrm{q}}^{+}, \mathcal{H}\right]$
[2.21c]
ih $\dot{\mathrm{T}}_{\underline{\mathrm{q}}}^{-}=\left[\mathrm{T}_{\underline{\mathrm{q}}}^{-}, \mathcal{H}\right]$
[2.21d]
ih $\dot{\mathrm{r}}_{-\mathrm{q}}^{+}=\left[\mathrm{T}_{-\mathrm{q}}^{+}, \mathcal{H}\right]$

assuming that the operators vary with time according to $\exp \left(-i \omega_{\underline{q}} t\right)$ or $\exp \left(-i \omega_{-q} t\right)$

The commutators of the two-ion terms of $\mathcal{K}$ may be worked out using the commutation relations [2.7], while the commutators of the single-ion terms are most easily found using the standard relations for tensor operators (Edmonds 1957, p.71).

$$
\left[s_{i}^{ \pm}, \widetilde{o}_{\ell m}\right]=[\ell(\ell+1)-m(m \pm 1)]^{\frac{1}{2}} \widetilde{o}_{\ell m \pm 1}
$$

$$
\left[s_{i}^{z}, \widetilde{\sigma}_{\ell m}\right]=m \widetilde{o}_{\ell m}
$$

In the case of spiral structures $\left(\underline{k}_{0} \neq 0\right)$, the hexagonal anisotropy gives rise to terms in the equations of motion involving the operators $s_{q+6 \underline{k}_{0}}^{ \pm}$and $s_{q-6 \underline{k}_{0}}^{ \pm}$. The equations of motion of these operators will, of course, couple to still others which differ in 
wave-vector by $\pm 6 \underline{k}_{0}$. Thus unless $6 \underline{k}_{0}$ is commensurate with a reciprocal lattice vector, modes of wave-vector $q$ are not well defined, as was first pointed out by Cooper et. al. (1962) . However, if ${ }^{B} \frac{6}{6}$ is weak relative to the axial crystal field constants, it should be a good approximation to neglect the terms in $\mathrm{s}_{\mathrm{q}_{ \pm}^{ \pm} 6 \mathrm{k}_{0}}$ in the equations of motion. We restrict ourselves to this case and therefore neglect all terms in ${ }_{6}^{6}$ for spiral structures. (This accounts for the presence of $\delta_{\underline{k}_{0}, 0}$ in the ${ }_{6}^{6}$ terms of equations [2.15] and [2.19].)

Following Englert (1960) and Brooks (1970) we linearized the equations of motion [2.21] by means of the same kind of approximations that were used in obtaining the stability conditions [2.19] and [2.20]. Having worked out the commutators in [2.21] and expressed them in terms of the Fourier-transformed operators, we replaced $\mathrm{S}_{\underline{\mathrm{q}}}^{\mathrm{z}}$ and $\mathrm{T}_{\mathrm{q}}^{\mathrm{Z}}$ by the first two terms of the Wortis expansion [2.9]. Then after commuting the operators $\mathrm{S}_{\underline{\mathrm{q}}}^{+}$and $\mathrm{T}_{\underline{\mathrm{q}}}^{+}$to the left of $\mathrm{S}_{\mathrm{q}}^{-}$and $\mathrm{T}_{\mathrm{q}}^{-}$and dropping terms in more than three operators, we Iinearized the remaining three-operator terms by the standard random phase approximations. The sums $\Sigma\left\langle\mathrm{S}_{\mathrm{q}^{+}}^{+} \mathrm{S}^{-}\right\rangle$and $\Sigma\left\langle\mathrm{T}_{\underline{q}^{+}}^{+} \underline{\mathrm{T}}^{-}\right\rangle$were expressed in terms of the reduced magnetization by means of [2.12], and powers of $\mathrm{m}$ were then obtained by the procedure exemplified by equation [B2]. Further details concerning these approximations are given in Appendix B.

As a result of these approximations the linearized equations of motion take the form,

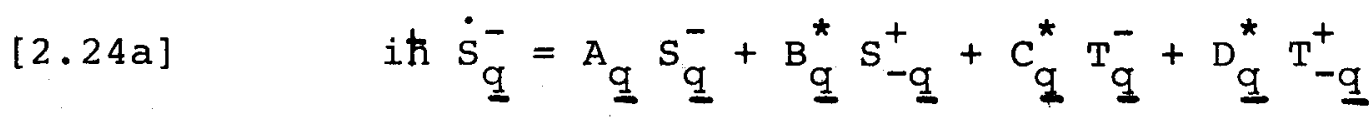




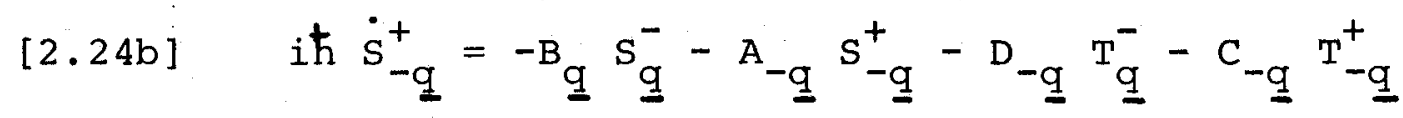

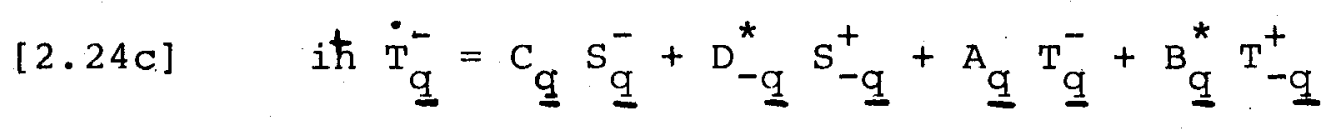

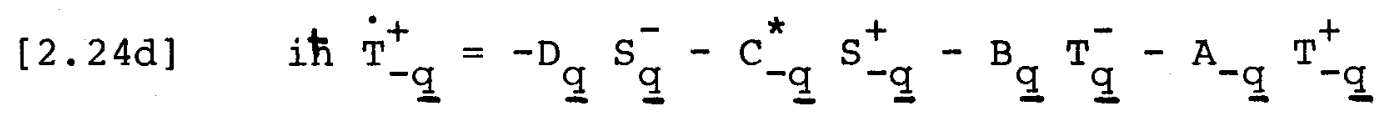

where,

$$
\begin{aligned}
A_{\underline{q}} & =S\left[\cos ^{2} \theta\left(J(0)+K(0)+J^{\prime}(0)+K^{\prime}(0)\right)+\sin ^{2} \theta\left(J\left(\underline{k}_{0}\right)+J^{\prime}\left(\underline{k}_{0}\right)\right)\right. \\
& \left.-\frac{1}{4}(1-\cos \theta)^{2} J\left(\underline{q}+\underline{k}_{0}\right)-\frac{1}{4}(1+\cos \theta)^{2} J\left(\underline{q}-\underline{k}_{0}\right)-\frac{1}{2} \sin ^{2} \theta(J(\underline{q})+K(\underline{q}))\right] m \\
& -\widetilde{B}(\theta)-21 B_{6}^{6} S(5 / 2) \sin ^{6} \theta \cos 6 \phi m^{20} \delta_{\underline{k}_{0}, 0}+\left(A_{m e}^{I}+A_{m e}^{I I}(\underline{q})\right) \delta_{\underline{k}_{0}, 0} \\
& +g \mu_{B} H_{\zeta} \cos \theta+g \mu_{B}\left(H_{\xi} \cos \phi+H_{n} \sin \phi\right) \sin \theta \delta_{\underline{k}_{0}, 0}
\end{aligned}
$$

[2.25]

$$
\begin{aligned}
B_{\underline{q}} & =S\left[\cos ^{2} \theta\left(J(0)+K(0)+J^{\prime}(0)+K^{\prime}(0)-J\left(\underline{k}_{0}\right)-J^{\prime}\left(\underline{k}_{0}\right)\right)\right. \\
& \left.+\frac{1}{4} \sin ^{2} \theta\left(J\left(\underline{q}+\underline{k}_{0}\right)+J\left(\underline{q}-\underline{k}_{0}\right)\right)-\frac{1}{2} \sin ^{2} \theta(J(\underline{q})+K(\underline{q}))\right] m \\
& -\widetilde{B}(\theta)+B_{6}^{6} S(5 / 2) \sin ^{4} \theta\left[\left(15+21 \cos ^{2} \theta\right) \cos 6 \phi-i 30 \cos \theta \sin 6 \phi\right] m^{20} \delta_{\underline{k}_{n}, 0} \\
& -\left(B_{m e}^{I}+B_{m e}^{I I}(\underline{q})\right) \delta_{\underline{k}_{0}, 0}+q \mu_{B} H_{\zeta} \cos \theta-g \mu_{B}\left(H_{\xi} \cos \phi+H_{n} \sin \phi\right) \cos \theta \cot \theta \delta_{\underline{k}_{0}, 0}
\end{aligned}
$$

$[2.26]$

$$
\begin{aligned}
C_{\underline{q}} & =-s\left[\frac{1}{4}(1-\cos \theta)^{2} J^{\prime}\left(\underline{q}+\underline{k}_{0}\right)+\frac{1}{4}(1+\cos \theta)^{2} J^{\prime}\left(\underline{q}-\underline{k}_{0}\right)\right. \\
& \left.+\frac{1}{2} \sin ^{2} \theta\left(J^{\prime}(\underline{q})+K^{\prime}(\underline{q})\right)\right] m+C_{m e}^{I I}(\underline{q}) \delta_{\underline{k}_{0}, 0}
\end{aligned}
$$




$$
\begin{aligned}
D_{q} & =s\left[\frac{1}{4} \sin ^{2} \theta\left(J^{\prime}\left(\underline{q}+\underline{k}_{0}\right)+J^{\prime}\left(\underline{q}-\underline{k}_{0}\right)\right)-\frac{1}{2} \sin ^{2} \theta\left(J^{\prime}(\underline{q})+K^{\prime}(\underline{q})\right)\right] m \\
& +D_{m e}^{I I}(\underline{q}) \delta_{\underline{k}_{0}, 0}
\end{aligned}
$$

$[2.28]$

and,

$$
\begin{aligned}
\widetilde{\mathrm{B}}(\theta) & =3 \widetilde{\mathrm{B}}_{2}^{0} \mathrm{~S}(1 / 2) \mathrm{P}_{2}^{0} \mathrm{~m}^{2}+10 \widetilde{\mathrm{B}}_{4}^{0} \mathrm{~S}(3 / 2) \mathrm{P}_{4}^{0} \mathrm{~m}^{9}+21 \widetilde{\mathrm{B}}_{6}^{0} \mathrm{~S}(5 / 2) \mathrm{P}_{6}^{0} \mathrm{~m}^{20} \\
& =\frac{\mathrm{d}}{\mathrm{dm}}\left(\widetilde{\mathrm{B}}_{2}^{0} \mathrm{~S}(1 / 2) \mathrm{P}_{2}^{0} \mathrm{~m}^{3}+\widetilde{\mathrm{B}}_{4}^{0} \mathrm{~S}(3 / 2) \mathrm{P}_{4}^{0} \mathrm{~m}^{10}+\widetilde{\mathrm{B}}_{6}^{0} \mathrm{~S}(5 / 2) \mathrm{P}_{6}^{0} \mathrm{~m}^{21}\right)
\end{aligned}
$$

[2.29]

As in equations [2.15], [2.19] and [2.20], $m$ stands for $m_{/}(T)$ when $\theta \neq \pi / 2$ and for $m(T)$ when $\theta=\pi / 2$, and $\mathrm{P}_{\ell}^{\mathrm{m}}$ is the associated Legendre function $\mathrm{P}_{\ell}^{\mathrm{m}}(\cos \theta)$. It should be mentioned that the stability condition [2.19] has been used in arriving at [2.26]. Finally, $A_{m e}^{I}$ and $B_{m e}^{I}$ are contributions from single-ion magnetoelastic terms while $A_{m e}^{I I}(\underline{q}), B_{m e}^{I I}(\underline{q}), C_{m e}^{I I}(\underline{q})$ and $D_{m e}^{I I}(\underline{q})$ are contributions from two-ion magnetoelastic terms, which are discussed in detail in section 3. These terms and the hexagonal anisotropy terms are to be included in [2.25]-[2.28] only when considering ferromagnetic structures, for the reasons given earlier. For the lowest order single-ion magnetoelastic terms contained in [2.1] one finds in the frozen lattice approximation,

$$
A_{m e}^{I}=\left(2 N S / c^{\gamma}\right) \frac{3}{4}\left(\widetilde{B}^{\gamma} S(1 / 2)\right)^{2} \sin ^{4} \theta m^{5}
$$

$$
+\left(2 \mathrm{NS} / \mathrm{C}^{\varepsilon}\right) \frac{3}{4}\left(\widetilde{\mathrm{B}}^{\varepsilon} \mathrm{S}(1 / 2)\right)^{2} \sin ^{2} 2 \theta \mathrm{m}^{5}
$$




$$
\begin{aligned}
\mathrm{B}_{\mathrm{me}}^{I} & =\left(2 \mathrm{NS} / \mathrm{C}^{\gamma}\right) \frac{1}{4}\left(\widetilde{\mathrm{B}}^{\gamma} \mathrm{S}(1 / 2)\right)^{2} \sin ^{2} \theta\left(3 \cos ^{2} \theta+1\right) \mathrm{m}^{5} \\
& +\left(2 \mathrm{NS} / \mathrm{C}^{\varepsilon}\right)\left(\widetilde{\mathrm{B}}^{\varepsilon} \mathrm{S}(1 / 2)\right)^{2} \cos ^{2} \theta\left(3 \cos ^{2} \theta-2\right) \mathrm{m}^{5}
\end{aligned}
$$

where $c^{\gamma}$ and $c^{\varepsilon}$ are elastic constants defined in [3.2] and [3.3] .

Although the expressions [2.25]-[2.28] are rather complicated, it can be seen that they possess a great deal of symmetry. Moreover they become much simpler in various special cases, such as,

(i) the limit $\mathrm{T}=0$, obtained by setting $\mathrm{m}=1$

(ii) ferromagnetic structures, obtained by setting $\underline{k}_{0}=0$

(iii) planar structures, obtained by setting $\theta=\frac{\pi}{2}$

(iv) the case of isotropic exchange only, obtained by setting $\mathrm{K}(\underline{\mathrm{q}})=0$ and $\mathrm{K}^{\prime}(\underline{\mathrm{q}})=0$

(v) the Bravais lattice approximation, obtained by setting $J^{\prime}(\underline{q})=K^{\prime}(\underline{q})=0$

\subsection{Solution of the Eigenvalue Problem}

The frequencies of spin wave modes of wave vectors $q$ and -q are found by requiring that the operators $\mathrm{S}_{\mathrm{q}^{\prime}}^{-} \mathrm{S}_{-\underline{\underline{q}}}^{+}, \mathrm{T}_{\underline{\mathrm{q}}}^{-}$and $\mathrm{T}_{-\underline{\mathrm{q}}}^{+}$ have the same time variation. Equation [2.24] then becomes an eigenvalue equation, the resulting determinantal equation having exactly the same form as that discussed by Lindgard, Kowalska and Laut (1967). In the present problem, since $J(-q)=J(q)$ and $J^{\prime}(-q)=$ $J^{\prime}(\underline{q})$ * with similar relations for $K(\underline{q})$ and $K^{\prime}(\underline{q})$, we have,

$[2.32 \mathrm{a}]$

$$
A_{-\underline{q}}-A_{q}=S \cos \theta\left[J\left(\underline{q}_{-}-\underline{k}_{0}\right)-J\left(\underline{q}+\underline{k}_{0}\right)\right]
$$

$$
\mathrm{B}_{-\underline{\mathrm{q}}}=\mathrm{B}_{\mathrm{q}}
$$


$[2.32 \mathrm{c}]$

$C_{-\underline{q}}^{*}-C_{\underline{q}}=s \cos \theta\left[J^{\prime}\left(\underline{q}-\underline{k}_{0}\right)-J^{\prime}\left(\underline{q}^{+} \underline{k}_{0}\right)\right]$

$[2.32 d]$

$$
\mathrm{D}_{-\underline{\underline{q}}}^{*}=\mathrm{D}_{\underline{\mathrm{q}}}
$$

It is instructive first to consider the eigenvalue problem in the Bravais lattice approximation. In this case the eigenvalues are

$$
\left.E_{\underline{q}}=\frac{1}{2}\left(A_{q}-\underline{A}_{-q}\right) \pm \frac{1}{2}\left(A_{\underline{q}}+\underline{A}_{-\underline{q}}\right)^{2}-4\left|B_{\underline{q}}\right|^{2}\right]^{\frac{1}{2}}
$$

which is the form found by Cooper et. al. (1962). It is clear from this expression and [2.32a] that in general $\mathrm{E}_{-q^{q}} \neq \mathrm{E}_{\mathrm{q}}$. In order that there be one positive eigenvalue for $\mathrm{E}_{\mathrm{q}}$ and one positive eigenvalue for $\mathrm{E}_{-\mathrm{q}}$ we require that

$$
\left[\left(\mathrm{A}_{\underline{q}}+\mathrm{A}_{-\underline{q}}\right)^{2}-4\left|\mathrm{~B}_{\underline{q}}\right|^{2}\right]^{\frac{1}{2}}>\left|\mathrm{A}_{\mathrm{q}}{ }^{-\mathrm{A}_{-q}}\right|
$$

Then the (positive) spin wave energies are

$$
\begin{aligned}
& E_{\underline{q}}=\frac{1}{2}\left(A_{q^{q}}-A_{-\underline{q}}\right)+\frac{1}{2}\left[\left(A_{\underline{q}}+A_{-\underline{q}}\right)^{2}-4\left|B_{q}\right|^{2}\right]^{\frac{1}{2}} \\
& E_{-\underline{q}}=-\frac{1}{2}\left(A_{\underline{q}}-A_{-\underline{q}}\right)+\frac{1}{2}\left[\left(A_{\underline{q}}+A_{-q}\right)^{2}-4\left|B_{\underline{q}}\right|^{2}\right]^{\frac{1}{2}}
\end{aligned}
$$

it being arbitrary which is called $\mathrm{E}_{\mathrm{q}}$ and which $\mathrm{E}_{-\mathrm{q}}$.

In solving the general problem of equation [2.24] we must allow $\mathrm{E}_{\mathrm{q}}$ and $\mathrm{E}_{-\mathrm{q}}$ to be unequal. One then has to solve a quartic equation of the form

$$
E_{\underline{q}}^{4}+f_{3}(\underline{q}) E_{\underline{q}}^{3}+f_{2}(\underline{q}) E_{\underline{q}}^{2}+f_{1}(\underline{q}) E_{\underline{q}}+f_{0}(\underline{q})=0
$$


By detailed examination it can be shown that $f_{2}(q)$ and $f_{0}(q)$ are even functions of $q$ while $f_{1}(q)$ and $f_{3}(g)$ are odd functions of $q$. It follows that if $\mathrm{E}_{\mathrm{q}}$ is a solution of [2.36], then $\left(-\mathrm{E}_{\underline{q}}\right)$ is a solution of the corresponding equation with $-\mathrm{q}$ replacing $q$ (Bar'yakhtar and Maleev, 1963). Thus among the roots of both equations there will be four positive roots, two of which belong to $\mathrm{E}_{\mathrm{g}}$ and two to $\mathrm{E}_{-\mathrm{q}}$. Unfortunately we cannot express these in a closed form, although, of course, they can be found numerically by standard methods.

However, a simplification occurs if $\mathrm{C}_{\mathrm{q}}$ and $\mathrm{D}_{\mathrm{q}}$ are both real. Thus, from [2.27] and [2.28], we need to know the wavevectors for which $J^{\prime}(q)$ and $K^{\prime}(q)$ are real. Let us rename the $\xi-$, $n$ - and $\zeta$-axes of the original coordinate system, denoting them as a-, b- and c-directions respectively. (The a-direction is toward a nearest-neighbour atom in the hexagonal plane.) The corresponding components of $q$ will be denoted $\left(q_{a}, q_{b}, q_{c}\right)$. Fig. 1 shows the fundamental one twenty-fourth part of the Brillouin zone with the usual labels for the symmetry points. TK is the a-direction, I'M is equivalent to the b-direction and $\Gamma A$ is the c-direction. Now since the crystal structure has mirror planes perpendicular to the a-direction and the c-direction it follows that $J^{\prime}(q)$ and $K^{\prime}(q)$ are real for any $q$-vector of the form $\left(q_{a}, 0, q_{c}\right)$. Thus they are both real for any $\mathrm{g}$ in the plane IKHA of Fig. 1. They are also real for any $\mathrm{g}$ in the plane $\mathrm{KMLH}$ since this plane is equivalent to an extension of the plane $\Gamma \mathrm{KHA}$ in the repeated zone scheme. Since $\underline{k}_{0}$ for spiral structures is along the c-direction, we conclude 


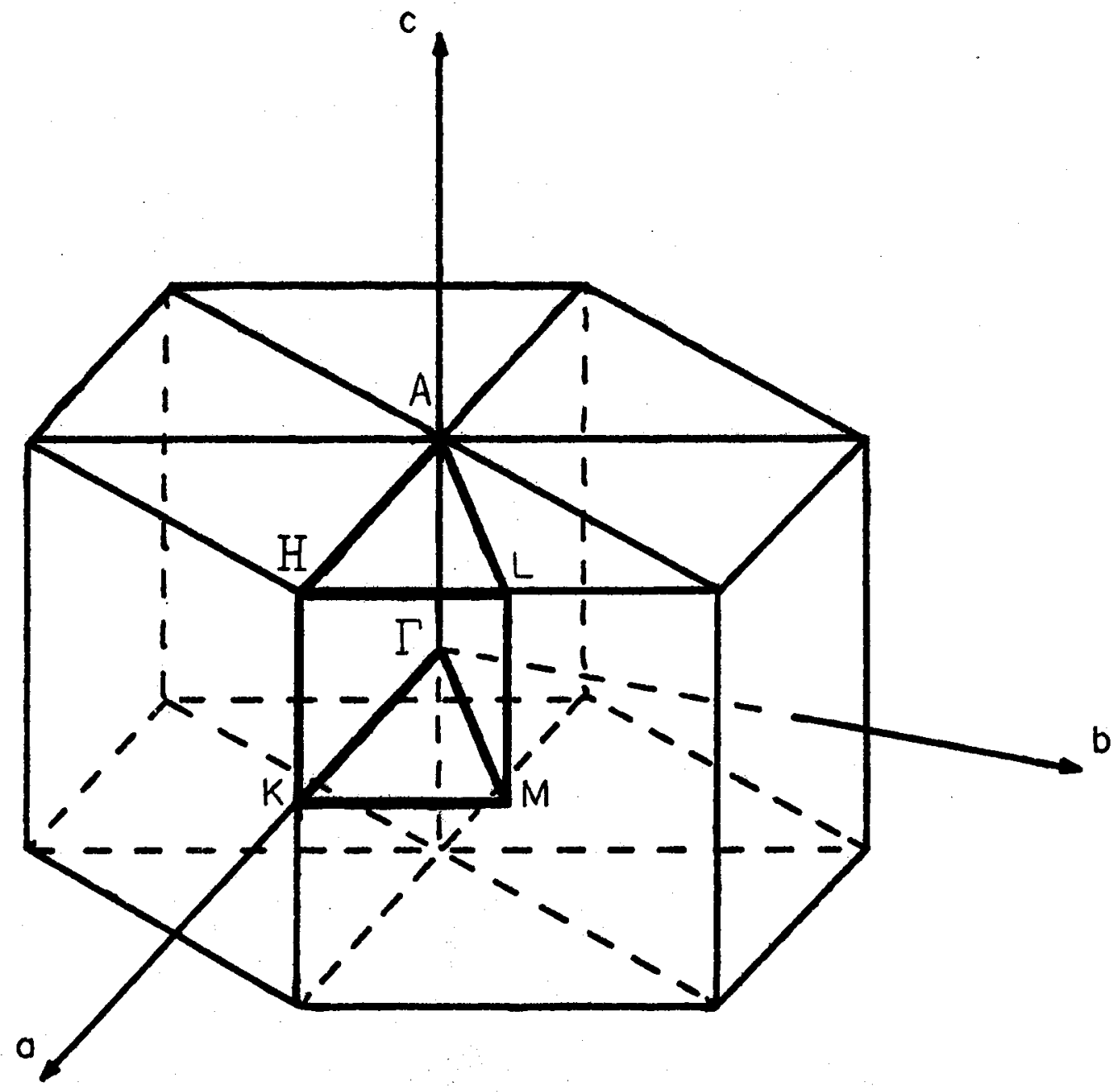

FIG. 1. First Brillouin zone for hap Structure 
that for all the structures considered in this paper $\mathrm{C}_{\mathrm{q}}$ and $\mathrm{D}_{\mathrm{q}}$ are real for q's lying in the planes $\Gamma \mathrm{KHA}$ and KMLH of Fig. 1 . Then it can be shown with the help of standard minipulations of the secular determinant of [2.24] that the solution is the same as the Bravais lattice solution [2.33] with $A_{q}$ replaced by $\mathrm{A}_{\mathrm{q}} \mathrm{FC}_{\mathrm{q}}$ and $\mathrm{B}_{\mathrm{q}}$ replaced by $\mathrm{B}_{\underline{q}}{ }^{\mathrm{D}} \mathrm{q}_{\mathrm{q}}$. Thus by the same reasoning which led to [2.35] we obtain the following expressions for the (positive) spin-wave energies:

$[2.37 a] \mathrm{E}_{\mathrm{q}}^{(\mathrm{i})}=\frac{1}{2}\left[\left(\mathrm{~A}_{\mathrm{q}} \mp \mathrm{C}_{\mathrm{q}}\right)-\left(\mathrm{A}_{-\mathrm{q}} \mp \mathrm{C}_{-q}\right)\right]+\frac{1}{2}\left\{\left[\left(\mathrm{~A}_{\mathrm{q}} \mp \mathrm{C}_{\mathrm{q}}\right)+\left(\mathrm{A}_{-\mathrm{q}} \mp \mathrm{C}-\underline{\mathrm{q}}\right)\right]^{2}-4\left(\mathrm{~B}_{\mathrm{q}} \mp \mathrm{D}_{\mathrm{q}}\right)^{2}\right\}^{\frac{1}{2}}$

$[2.37 b] E_{-q}^{(i)}=-\frac{1}{2}\left[\left(A_{q} \mp C_{q}\right)-\left(A_{-q} \mp C_{-q}\right)\right]+\frac{3}{2}\left\{\left[\left(A_{q} \mp C_{q}\right)+\left(A_{-q} \mp C_{-q}\right)\right]^{2}-4\left(B_{q} \mp D_{q}\right)^{2}\right\}^{\frac{1}{2}}$

The lower sign gives the lower spin-wave branch (labelled $i=1$ ) while the upper sign gives the upper branch $(i=2)$. We note that when $\mathrm{C}_{\mathrm{q}}$ and $\mathrm{D}_{\mathrm{q}}$ are real it can be shown from [2.37] that the Bravais lattice solution [2.35] correctly gives the lower spin-wave branch provided that the lattice sums $J(q)$ and $K($ g) that occur in [2.25] and [2.26] are taken to include all atoms, not just those on the same sublattice. Furthermore, as is well known, the upper mode in the c-direction can be obtained by extending the Bravais lattice solution in the double zone scheme. The same device cannot be used, however, in the a-direction. In addition to the case just described, analytic solutions may be obtained under other special conditions. From [2.32a] and $[2.32 \mathrm{c}]$ and the structure of $\mathrm{J}(\underline{q})$ and $\mathrm{J}^{\prime}(\underline{q})$ it is clear that 
$A_{-q}=A_{q}$ and $c_{-q}^{*}=C_{q}$ if any of the following conditions are satisfied:

(i) q lies in the basal plane (ie. $\underline{q}$ is perpendicular to $\underline{k}_{0}$ )

(ii) $\quad \underline{k}_{0}=0$

(iii) $\theta=\pi / 2$.

Then it can be shown that $f_{1}(q)$ and $f_{3}(q)$ are zero and equation [2.36] reduces to a quadratic equation in $\mathrm{E}_{q}^{2}$. The (positive) solutions are (Lindgărd et. al. 1967),

$$
\begin{aligned}
& E_{\underline{q}}^{(i)}=\left(R_{\underline{q}} \mp \sqrt{S_{q}}\right)^{\frac{3}{2}} \\
& R_{\underline{q}}=A_{\underline{q}}^{2}-\left|B_{\underline{q}}\right|^{2}+\left|C_{\underline{q}}\right|^{2}-\left|D_{\underline{q}}\right|^{2} \\
& \dot{S}_{q}=2\left|A_{q} C_{q}-B^{*}{ }^{*} D_{\underline{q}}\right|^{2}+2\left|A_{\underline{q}} C^{C}-\underline{q}^{-B}{ }^{*}{ }^{D}-\underline{q}\right|^{2}
\end{aligned}
$$

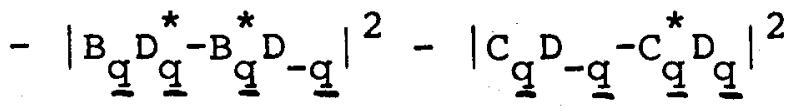

$$
\begin{aligned}
& =\left|2 A_{q} C_{q}-\left(B_{q}+B_{q}^{*}\right) D_{q}\right|^{2}-\left|C_{q} D_{q}^{*}-C^{*}{ }^{*}{ }^{q}\right|^{2}
\end{aligned}
$$

where $i$ again labels the spin wave branches corresponding to the $\mp$ signs. The property $\mathrm{D}_{-\mathrm{q}^{\mathrm{q}}}=\mathrm{D}_{\underline{\mathrm{q}}}^{*}$ has been used to obtain the last line of [2.39b]. It is worth emphasizing that this solution is valid for all q-vectors in all spin structures under consideration with one exception, -- the case of a conical spiral with $q$ having a component parallel to $\underline{k}_{0}$. (However, in this case one still has the solution [2.37] for q's in the planes TKHA or KMLH of Fig. 1.) Finally, if the conditions for the solution [2.37] and those for the solution [2.38]-[2.39] are satisfied simultaneously 
32

(ie. $A_{-q}=A_{q}, C_{-q}=C_{q}$ and both $C_{q}$ and $D_{q}$ are real) then both solusions simplify to

$[2.40]$

$$
\mathrm{E}_{\underline{\mathrm{q}}}^{(\mathrm{i})}=\left(\mathrm{A}_{\mathrm{q}} \mp \mathrm{C}_{\mathrm{q}}+\left|\mathrm{B}_{\underline{q}} \mp \mathrm{D}_{\mathrm{q}}\right|\right)^{\frac{1}{2}}\left(\mathrm{~A}_{\mathrm{q}} \mp \mathrm{C}_{\mathrm{q}}-\left|\mathrm{B}_{\underline{\mathrm{q}}} \mp \mathrm{D}_{\mathrm{q}}\right|\right)^{\frac{1}{2}}
$$




\section{CHAPTER 3}

\section{MAGNETOELASTIC EFFECTS OF HIGHER ORDER}

Turov and Shavrov (1965) and Cooper (1968a) have demonstrated how magnetostrictive terms in the Hamiltonian contribute to the spin-wave energies. In Cooper's paper the lowest order single-ion magnetoelastic terms (quadratic in $s_{i}^{\xi}, s_{i}^{n}$ and $s_{i}^{\zeta}$ ) are treated microscopically following the theory of callen and Callen (1965), but the higher order terms (fourth order in $s_{i}^{\xi}$, $s_{i}^{\eta}$ and $s_{i}^{\zeta}$ ) are treated macroscopically.

The present formulation is entirely microscopic. All possible single-ion magnetoelastic terms linear in the strains and up to fourth order in the spin operators $s_{i}^{\xi}, s_{i}^{n}$ and $s_{i}^{\zeta}$ are written down and the form of the contribution that each makes to the spinwave energies and the conditions for stable equilibrium is determined. It will be found that some of these terms have the same form as the crystal field terms while others have different forms. The contributions of two-ion magnetoelastic terms linear in the strains and second order in the spin operators are also examined.

\section{I The Theory of Callen and Callen}

Our description of magnetostrictive effects is based on the general theory of Callen and Callen (1965). The homogeneous strains which transform according to the irreducible representations $\Gamma_{\alpha}, \Gamma_{\gamma}$ and $\Gamma_{\varepsilon}$ of the (chemical) point group of the hcp lattice are, in the notation of Callen and Callen and of Cooper (1968),

$[3.1 \mathrm{a}]$

$$
\varepsilon^{\alpha, 1}=\varepsilon_{\xi \xi}+\varepsilon_{n \eta}+\varepsilon_{\zeta \zeta}
$$


[3.1b]

$\varepsilon^{\alpha, 2}=\varepsilon_{\zeta \zeta}-\frac{1}{3} \varepsilon^{\alpha, 1}$

[3.1c]

$\varepsilon_{1}^{\gamma}=\frac{1}{2}\left(\varepsilon_{\xi \xi}-\varepsilon_{n \eta}\right)$

[3.1d]

$\varepsilon_{2}^{\gamma}=\varepsilon_{\xi n}$

[3.1e]

$\varepsilon_{1}^{\varepsilon}=\varepsilon_{n \zeta}$

[3.1f]

$\varepsilon_{2}^{\varepsilon}=\varepsilon_{\xi \zeta}$

where $\varepsilon_{i j}=\frac{1}{2}\left[\left(\partial u_{i} / \partial r_{j}\right)+\left(\partial u_{j} / \partial r_{i}\right)\right], i, j=\xi, n, \zeta$ and $\underline{u}$ is the displacement of a point relative to its equilibrium position. Then the elastic energy associated with the homogeneous strains is

$$
\begin{aligned}
\mathcal{H}_{e} & =\frac{1}{2} c_{1}^{\alpha}\left(\varepsilon^{\alpha, 1}\right)^{2}+c_{12} \varepsilon^{\alpha, 1} \varepsilon^{\alpha, 2}+\frac{1}{2} c_{2}^{\alpha}\left(\varepsilon^{\alpha, 2}\right)^{2} \\
& +\frac{1}{2} c^{\gamma}\left[\left(\varepsilon_{1}^{\gamma}\right)^{2}+\left(\varepsilon_{2}^{\gamma}\right)^{2}\right]+\frac{1}{2} c^{\varepsilon}\left[\left(\varepsilon_{1}^{\varepsilon}\right)^{2}+\left(\varepsilon_{2}^{\varepsilon}\right)^{2}\right] .
\end{aligned}
$$

Following callen and callen, we omit the nonhomogeneous strains or phonon modes from the Hamiltonian, so that $\mathcal{H}_{e}$ is purely classical. The $c^{\Gamma}$ 's are the elastic stiffness constants which are related to the five independent Cartesian elastic constants by

[3.3a]

$$
c_{1}^{\alpha}=\frac{1}{9}\left(2 c_{11}+2 c_{12}+4 c_{13}+c_{33}\right)
$$

[3. 3b]

$$
c_{2}^{\alpha}=\frac{1}{2}\left(c_{11}+c_{12}-4 c_{13}+2 c_{33}\right)
$$


$[3.3 c]$

$$
c_{12}^{\alpha}=\frac{1}{3}\left(-c_{11}-c_{12}+c_{13}+c_{33}\right)
$$

[3. 3d]

$$
c^{\gamma}=2\left(c_{11}-c_{12}\right)
$$

[3.3e]

$$
\mathrm{c}^{\varepsilon}=4 \mathrm{c}_{44}
$$

Callen and Callen consider two types of magnetoelastic terms linear in the strains. The first is single-ion terms formed by taking products of the symmetry strains and spin functions belonging to the same irreducible representation. Instead of adopting their expression, as in our equation [2.1], we shall express the spin functions in terms of the operator equivalents $\tilde{O}_{\ell m}$ introduced in section 2.1. For $\ell=2$ and 4 it can be shown that the linear combinations

$$
\begin{aligned}
& \text { [3.4a] } \\
& \tilde{\delta}_{\ell m}{ }^{+}=\frac{1}{2}\left(\gamma_{\ell m}+\tilde{\sigma}_{\ell-m}\right) \\
& \text { [3.4b] } \\
& \tilde{\sigma}_{\ell m}{ }^{-}=\frac{1}{2 i}\left(\tilde{\sigma}_{\ell m}-\tilde{O}_{\ell-m}\right)
\end{aligned}
$$

transform according to particular irreducible representations of the point group $\overline{6} \mathrm{~m} 2$. (Odd powers of the spin operators do not occur in the Hamiltonian because they are not invariant under time reversal.) In terms of these functions we can write the single-ion magnetoelastic terms as,

$$
\text { [3.5a] } \quad \mathcal{L}_{\text {me }}^{I}=\sum_{i} \mathcal{H}_{\mathrm{me}}^{I}(i)
$$

$\mathcal{H}_{\text {me }}^{I}(i)=-M_{20}^{\alpha, 1} \varepsilon^{\alpha, 1} \gamma_{20}-M_{20}^{\alpha, 2} \varepsilon^{\alpha, 2} \gamma_{20}-M_{2 I}^{\varepsilon}\left[\varepsilon_{1}^{\varepsilon}\left(i \gamma_{21}^{+}\right)+\varepsilon_{2}^{\varepsilon}\left(-i \gamma_{21}^{-}\right)\right]$ 


$$
\begin{aligned}
&-M_{22}^{\gamma}\left(\varepsilon{ }_{1}^{\gamma} \widetilde{O}_{22}^{+}+\varepsilon_{2}^{\gamma} \tilde{O}_{22}^{-}\right)-M_{40}^{\alpha, 1} \varepsilon^{\alpha, 1} \tilde{O}_{40}-M_{40}^{\alpha, 2} \varepsilon^{\alpha, 2} \tilde{O}_{40} \\
&-M_{41}^{\varepsilon}\left[\varepsilon_{1}^{\varepsilon}\left(i \tilde{O}_{41}^{+}\right)+\varepsilon_{2}^{\varepsilon}\left(-i \tilde{O}_{41}^{-}\right)\right]-M_{42}^{\gamma}\left(\varepsilon_{1}^{\gamma} \gamma_{42}^{+}+\varepsilon_{2}^{\gamma \gamma} \tilde{O}_{42}^{-}\right) \\
& {[3.5 b]-M_{44}^{\gamma}\left(\varepsilon_{1}^{\gamma} \gamma_{44}^{+}-\varepsilon{ }_{2}^{\gamma} \gamma_{44}^{-}\right) . }
\end{aligned}
$$

The operators $\tilde{\mathrm{O}}_{43}^{+}$and $\tilde{\mathrm{O}}_{43}^{-}$do not appear in this expression as they transform according to irreducible representations of $\overline{6} \mathrm{~m} 2$ other than $\Gamma_{\alpha}, \Gamma_{\gamma}$ and $\Gamma_{\varepsilon}$. The $M_{\ell m}^{\Gamma}$ 's are phenomenological magnetoelastic coupling constants introduced in the same spirit as the crystal field parameters $\widetilde{B}_{\ell}^{m}$. Those for $l=2$ are related to the callen and callen constants by

$$
\begin{array}{ll}
\text { [3.6a] } & \widetilde{\mathrm{B}}_{12}^{\alpha}=(3)^{\frac{1}{2}} \mathrm{M}_{20}^{\alpha, 1}, \widetilde{\mathrm{B}}_{22}^{\alpha}=(3)^{\frac{1}{2}} \mathrm{M}_{20}^{\alpha, 2} \\
{[3.6 \mathrm{~b}]} & \widetilde{\mathrm{B}}^{\gamma}=\left(\frac{3}{2}\right)^{\frac{1}{2}} \mathrm{M}_{22}^{\gamma}, \widetilde{\mathrm{B}^{\varepsilon}}=\left(\frac{3}{2}\right)^{\frac{1}{2}} \mathrm{M}_{21}^{\varepsilon} .
\end{array}
$$

The two-ion magnetoelastic terms linear in the strains and second order in the spin operators are, in the notation of callen and Callen,

$$
\begin{aligned}
& {[3.7 \text { a] }} \\
& \mathcal{H}_{\text {me }}^{I I}=\sum_{i<j} \mathcal{H}_{\text {me }}^{I I}(i, j) \\
& \mathcal{H}_{m e}^{I I}(i, j)=-\widetilde{D}_{11 i j}^{\alpha} \varepsilon^{\alpha,{ }^{\prime}} \underline{S}_{i} \cdot \underline{S}_{j}-\widetilde{D}_{12 i j}^{\alpha} \varepsilon^{\alpha, 1}(\sqrt{3} / 2)\left(S_{i}^{\zeta} S_{j}^{\zeta}-\frac{1}{3} \underline{S}_{i} \cdot \underline{S}_{j}\right) \\
& -\widetilde{D}_{21 i j}^{\alpha} \varepsilon^{\alpha, 2} \underline{S}_{i} \cdot \underline{S}_{j}-\widetilde{D}_{22}^{\alpha} i j \varepsilon^{\alpha, 2}(\sqrt{3} / 2)\left(S_{i}^{\zeta} S_{j}^{\zeta}-\frac{1}{3} S_{i} \cdot \underline{S}_{j}\right) \\
& -\tilde{D}_{i j}^{\gamma}\left[\varepsilon_{1}^{\gamma} \frac{1}{2}\left(S_{i}^{\xi} S_{j}^{\xi}-S_{i}^{n} S_{j}^{n}\right)+\varepsilon_{2}^{\gamma} \frac{1}{2}\left(S_{i}^{\xi} S_{j}^{n}+S_{i}^{n} s_{j}^{\xi}\right)\right]
\end{aligned}
$$


$[3.7 b]-\tilde{D}_{i j}^{\varepsilon}\left[\varepsilon_{1}^{\varepsilon_{1}}\left(S_{i}^{n} s_{j}^{\zeta}+s_{i}^{\zeta} s_{j}^{n}\right)+\varepsilon_{2}^{\varepsilon} \frac{1}{2}\left(S_{i}^{\xi} s_{j}^{\zeta}+s_{i}^{\zeta} S_{j}^{\xi}\right)\right]$.

The equilibrium values of the strains are found by minimizing $\mathcal{H}_{e}+\mathcal{H}_{\text {me }}^{I}+\mathcal{H}_{m e}^{I I}$, and this can be carried out in two limiting approximations, which will be discussed separately. It should be mentioned before going further that the assumption of homogeneous strains requires that the magnetization be uniform, and this means that the above forms of the magnetoelastic coupling are not applicable to spiral structures (although particular terms may be in special cases). Therefore we shall consider only ferromagnetic structures in what follows.

\subsection{The Frozen Lattice Approximation}

If it is assumed that the strains are "frozen" at their equilibrium values, which do not vary with time, one obtains the frozen lattice approximation discussed by Turov and Shavrov (1965) and cooper (1968a). The spin functions in [3.5] and [3.7] are replaced by thermal averages which may be regarded as containing the effect of averaging with respect to time. Then by minimizing $\mathcal{H}_{e}+\mathcal{H}_{\text {me }}^{I}+\mathcal{H}_{\text {me }}^{I I}$ one can obtain the equilibrium strains $\bar{\varepsilon}^{\alpha, 1}, \bar{\varepsilon}^{\alpha, 2}$, $\bar{\varepsilon}_{1}^{\gamma}, \bar{\varepsilon}_{2}^{\gamma}, \bar{\varepsilon}_{1}^{\varepsilon}$ and $\bar{\varepsilon}_{2}^{\varepsilon}$. It is apparent that with these expressions substituted into [3.5] and [3.7] certain terms have exactly the same dependence on spin operators as crystal field terms or isotropic and anisotropic exchange terms. We can drop these terms from $\mathcal{H}_{\text {me }}^{I}$ and $\mathcal{H}_{\text {me }}^{\text {II by redefining, }}$

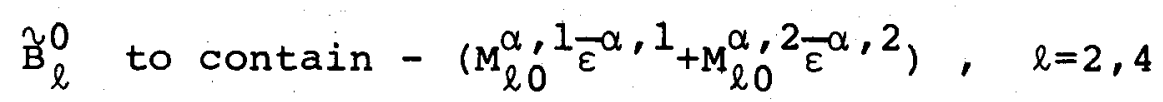




$$
\begin{aligned}
& J_{i j} \text { to contain }\left[\tilde{D}_{11}^{\alpha} i j-(2 \sqrt{3})^{-\frac{1}{2}} \hat{D}_{12}^{\alpha} i j\right] \bar{\varepsilon}^{\alpha, 1} \\
& +\left[\tilde{D}_{21 i j}^{\alpha}-(2 \sqrt{3})^{-\frac{1}{2}} \tilde{D}_{22}^{\alpha} i j\right]^{E^{\alpha, 2}}
\end{aligned}
$$

[3.8] $\mathrm{K}_{i j}$ to contain $(\sqrt{3} / 2)\left(\tilde{\mathrm{D}}_{12}^{\alpha} i j^{\bar{\varepsilon}^{\alpha, 1}}+\tilde{\mathrm{D}}_{22}^{\alpha} i j^{\left.\bar{\varepsilon}^{\alpha, 2}\right)}\right.$.

It is of interest to note that these magnetoelastic contributions to the parameters are temperature dependent since the equilibrium strains $\bar{\varepsilon}^{\alpha, 1}$ and $\bar{\varepsilon}^{\alpha, 2}$ consist of terms which vary as $\mathrm{m}^{2}, \mathrm{~m}^{3}$ and $\mathrm{m}^{10}$ when evaluated by the usual approximations.

For the magnetoelastic terms that remain, after evaluating the thermal averages with the aid of the usual approximations, one obtains for the equilibrium strains

[3.9a] $\bar{\varepsilon} \gamma \equiv \bar{\varepsilon}_{1}^{\gamma}+i \bar{\varepsilon}_{2}^{\gamma}$

$$
\bar{\varepsilon}^{\gamma}=\left(2 \mathrm{NS} / \mathrm{C}^{\gamma}\right)\left[\left[\mathrm{M}_{22}^{\gamma} \mathrm{S}(1 / 2) \mathrm{m}^{3}+(2 / 3)^{\frac{1}{2} \tilde{\mathrm{D}}{ }^{\gamma}} \mathrm{Sm}^{2}\right] \tilde{Y}_{22}\right.
$$

$\left.[3.9 b]+\left(M_{42}^{\gamma} \Psi_{42}+M_{44}^{\gamma} \Psi_{4-4}\right) \mathrm{S}(3 / 2) \mathrm{m}^{10}\right\}$

[3.9c] $\quad \bar{\varepsilon}^{\varepsilon} \equiv \bar{\varepsilon}_{2}^{\varepsilon}+i \bar{\varepsilon}_{1}^{\varepsilon}$

$$
\bar{\varepsilon}^{\varepsilon}=\left(2 \mathrm{NS} / \mathrm{C}^{\varepsilon}\right) \quad\left[\left[\mathrm{M}_{21}^{\varepsilon} \mathrm{S}(1 / 2) \mathrm{m}^{3}+(2 / 3)^{\frac{1}{2} \tilde{D}} \mathrm{~S}^{2}\right]\left(-\widetilde{Y}_{21}\right)\right.
$$

[3.9d] $\left.\quad+\mathrm{M}_{4 I}^{\varepsilon}\left(-\vec{Y}_{4 I}\right) \mathrm{S}(3 / 2) \mathrm{m}^{10}\right\}$

where the symbol $\widetilde{Y}_{\ell m}$ stands for an unnormalized spherical harmonic which is related to the usual normalized spherical harmonic $\mathrm{Y}_{\ell \mathrm{m}}$ by the relation

$$
\text { [3.10] } \breve{Y}_{\ell m}(\theta, \phi)=[4 \pi /(2 \ell+1)]^{\frac{1}{2}} \mathrm{Y}_{\ell m}(\theta, \phi) .
$$


(Thus the $\widetilde{Y}_{\text {lm }}$ have the same constant factors as the Racah operator equivalents $\widetilde{o}_{\ell m}$. ) Note that our definition of the $\widetilde{Y}_{\ell m}$ or the $Y_{\ell m}$ (Edmonds 1957) contains a factor $(-1)^{\mathrm{m}}$ for $\mathrm{m}>0$. The symbols $\widetilde{\mathrm{D}}^{\gamma}$ and $\widetilde{D}^{\varepsilon}$ in [3.9b] and [3.9d] remain to be defined. It is convenient to introduce at this point the definitions

[3.11a]

$[3.11 b]$

$$
\begin{aligned}
\widetilde{D}^{\gamma}(\underline{q}) & =\sum_{i}, \widetilde{D}_{i j}^{\gamma}, \exp \left[i \underline{q} \cdot\left(\underline{r}_{i}-\underline{r}_{i},\right)\right] \\
\widetilde{D}^{\prime}(\underline{q}) & =\sum_{j} \widetilde{D}_{i j}^{\gamma} \exp \left[i \underline{q} \cdot\left(\underline{r}_{i}-\underline{r}_{j}\right)\right]
\end{aligned}
$$

with similar definitions for $\widetilde{D}^{\varepsilon}(q)$ and $\widetilde{D}^{\varepsilon^{\prime}}(q)$. Here $i$ ' runs over the same sublattice as $i$ while $j$ runs over the other sublattice. Then the constants $\widetilde{\mathrm{D}}^{\gamma}$ and $\widetilde{\mathrm{D}}^{\varepsilon}$ are defined by
$[3.12 a]$
$\widetilde{D}^{\gamma}=\frac{1}{2}\left[D^{\gamma}(0)+D^{\gamma^{\prime}}(0)\right]$
$[3.12 b]$
$\widetilde{D}^{\varepsilon}=\frac{1}{2}\left[D^{\varepsilon}(0)+D^{\varepsilon^{\prime}}(0)\right]$.

Each of the terms in [3.9] is easily identified with one of the terms in $\mathcal{H}_{\text {me }}^{I}$ or $\mathcal{H}_{\mathrm{me}}^{\mathrm{II}}$. It can be seen that the single-ion magnetoelastic terms follow the $\ell(\ell+1) / 2$ power law while the twoion terms vary as $\mathrm{m}^{2}$ (Callen and Callen 1966). We note that the equilibrium strains depend on the direction of the magnetization through the spherical harmonics $\widetilde{Y}_{\ell m}(\theta, \phi)$ with $\theta$ and $\phi$ as in equation [2.2].

It is useful to express the equilibrium strains in terms of the saturation magnetostriction constants. Instead of using the ones defined by Callen and Callen (see their equation [4.27]), we find it more convenient for the present discussion to define a 
set of constants relative to an expansion in spherical harmonics:

$$
\begin{aligned}
\delta l / l & =\left(\lambda_{00}^{\alpha, 1} \widetilde{Y}_{00}+\lambda_{20}^{\alpha, 1} \widetilde{Y}_{20}+\lambda_{40}^{\alpha, 1} \widetilde{Y}_{40}\right) \\
& +\left(\lambda_{00}^{\alpha, 2} \widetilde{Y}_{00}+\lambda_{20}^{\alpha, 2} \widetilde{Y}_{20}+\lambda_{40}^{\alpha, 2} \widetilde{Y}_{40}\right)\left(\beta_{\zeta}^{2}-\frac{1}{3}\right) \\
& +\left(\lambda \gamma_{22}^{\gamma} \operatorname{Re}\left\{\widetilde{Y}_{22}\left(\beta_{\xi}-i \beta_{\eta}\right)^{2}\right\}+\lambda_{42}^{\gamma} \operatorname{Re}\left\{\widetilde{Y}_{42}\left(\beta_{\xi}-i \beta_{\eta}\right)^{2}\right\}\right. \\
& \left.+\lambda{ }_{44}^{\gamma} \operatorname{Re}\left\{\widetilde{Y}_{4-4}\left(\beta_{\xi}-i \beta_{\eta}\right)^{2}\right\}\right)
\end{aligned}
$$

$[3.13]+\left(\lambda_{21}^{\varepsilon} \operatorname{Re}\left\{2\left(-\widetilde{Y}_{21}\right)\left(\beta_{\xi}-i \beta_{\eta}\right) \beta_{\zeta}\right\}+\lambda_{41}^{\varepsilon} \operatorname{Re}\left\{2\left(-\widetilde{Y}_{41}\right)\left(\beta_{\xi}-i \beta_{n}\right) \beta_{\zeta}\right\}\right)$

Here $\delta l / l$ is the fractional change in length of the crystal measured in the direction $\left(\beta_{\xi}, \beta_{\eta}, \beta_{\zeta}\right)$ when the magnetization is in the direction $(\theta, \phi)$. The magnetostriction constants $\lambda^{\gamma}$ and $\lambda^{\varepsilon}$ employed by Callen and Callen and by Clark, De Savage and Bozorth are related to these by

$$
\lambda^{\gamma}=(3 / 2)^{\frac{1}{2}} \lambda_{22}^{\gamma}, \quad \lambda^{\varepsilon}=(3 / 2)^{\frac{1}{2}} \lambda_{21}^{\varepsilon}
$$

The magnetostriction constants defined by Mason (1954) and employed

${ }^{1}$ Our terms in $\lambda_{44}^{\gamma}$ do not correspond solely to Mason's terms in $A$; in addition they contribute an amount $\lambda_{44}^{\gamma}(70)^{1 / 2} / 16$ to $D$ and $-\lambda_{44}^{\gamma}(70)^{1 / 2} / 16$ to $\mathrm{E}$.

by Rhyne and Legvold (1965) and Rhyne and Clark (1967) are given 
by

[3.15a]

$A=-\left[(70)^{\frac{1}{2}} / 8\right] \lambda_{44}^{\gamma}$

$[3.15 b]$

$B=\left[7(10)^{\frac{1}{2}} / 8\right] \lambda_{42}^{\gamma}$

[3.15c]

$C=\left[(6)^{\frac{1}{2}} / 4\right] \lambda_{22}^{\gamma}-\left[(10)^{\frac{1}{2}} / 8\right] \lambda_{42}^{\gamma}$

$[3.15 d]$

$\mathrm{H}=(6)^{\frac{1}{2}} \lambda_{2 I}^{\varepsilon}-\left[3(5)^{\frac{1}{2}} / 2\right] \lambda_{4 I}^{\varepsilon}$

$[3.15 e]$

$I=\left[7(5)^{\frac{1}{2}} / 2\right] \lambda_{41}^{\varepsilon}$

Note that these vary with temperature through the reduced magnetization according to equation [3.19] below.

The connection between the magnetostriction constants and the equilibrium strains is established through the relation (see, for example, Birss (1964), p.184)

$$
\delta \ell / \ell=\sum_{\mu, \nu} \bar{\varepsilon}_{\mu \nu} \beta_{\mu}^{\beta} \nu \quad \mu, \nu=x, y, z
$$

which may be rewritten in terms of the symmetry strains [3.1] as

$$
\delta \ell / \ell=\frac{1}{3} \bar{\varepsilon}^{\alpha, 1}+\frac{3}{2} \bar{\varepsilon}^{\alpha, 2}\left(\beta_{\zeta}^{2}-\frac{1}{3}\right)+\bar{\varepsilon}_{1}^{\gamma}\left(\beta_{\xi}^{2}-\beta_{\eta}^{2}\right)+2 \bar{\varepsilon}_{2}^{\gamma} \beta_{\xi} \beta_{\eta}
$$

[3.17]

$$
+2 \bar{\varepsilon}_{1}^{\varepsilon} \beta_{\eta}^{\beta}{ }_{\zeta}+2 \bar{\varepsilon}_{2}^{\varepsilon} \beta_{\xi}^{\beta} \zeta
$$

Comparing [3.13] and [3.17] we find

$[3.18 a]$

$$
\bar{\varepsilon}^{\alpha, I}=3\left(\lambda_{00}^{\alpha, l} \widetilde{Y}_{00}+\lambda_{20}^{\alpha, l} \widetilde{Y}_{20}+\lambda_{40}^{\alpha, l} \widetilde{Y}_{40}\right)
$$

[3.18b]

$$
\bar{\varepsilon}^{\alpha, 2}=(2 / 3)\left(\lambda_{00}^{\alpha, 2} \widetilde{Y}_{00}+\lambda_{20}^{\alpha, 2} \widetilde{Y}_{20}+\lambda_{40}^{\alpha, 2} \widetilde{Y}_{40}\right)
$$




$$
\begin{array}{ll}
{[3.18 \mathrm{c}]} & \bar{\varepsilon}_{1}^{\gamma}=\lambda_{22}^{\gamma} \operatorname{Re}\left\{\widetilde{\mathrm{Y}}_{22}\right\}+\lambda_{42}^{\gamma} \operatorname{Re}\left\{\widetilde{\mathrm{Y}}_{42}\right\}+\lambda_{44}^{\gamma} \operatorname{Re}\left\{\widetilde{Y}_{4-4}\right\} \\
{[3.18 \mathrm{~d}]} & \bar{\varepsilon}_{2}^{\gamma}=\lambda_{22}^{\gamma} \operatorname{Im}\left\{\widetilde{Y}_{22}\right\}+\lambda_{42}^{\gamma} \operatorname{Im}\left\{\widetilde{Y}_{42}\right\}+\lambda_{44}^{\gamma} \operatorname{Im}\left\{\widetilde{Y}_{4-4}\right\} \\
{[3.18 \mathrm{e}]} & \bar{\varepsilon}_{1}^{\varepsilon}=\lambda_{21}^{\varepsilon} \operatorname{Im}\left\{\left(-\widetilde{Y}_{21}\right)\right\}+\lambda_{41}^{\varepsilon} \operatorname{Im}\left\{\left(-\widetilde{Y}_{41}\right)\right\} \\
{[3.18 f]} & \bar{\varepsilon}_{2}^{\varepsilon}=\lambda_{21}^{\varepsilon} \operatorname{Re}\left\{\left(-\widetilde{Y}_{21}\right)\right\}+\lambda_{41}^{\varepsilon} \operatorname{Re}\left\{\left(-\widetilde{Y}_{41}\right)\right\}
\end{array}
$$

These are the required relations expressing the equilibrium strains in terms of the experimentally measurable magnetostriction constants.

$$
\text { The magnetostriction constants are related to the }
$$

phenomenological magnetoelastic coupling constants by

$$
\begin{array}{ll}
{[3.19 \mathrm{a}]} & \lambda_{22}^{\gamma}=\left(2 \mathrm{NS} / \mathrm{C}^{\gamma}\right)\left[\mathrm{M}_{22}^{\gamma} \mathrm{S}(1 / 2) \mathrm{m}^{3}+(2 / 3)^{\frac{1}{2}} \widetilde{\mathrm{D}}^{\gamma} \mathrm{Sm}^{2}\right] \\
{[3.19 \mathrm{~b}]} & \lambda_{42}^{\gamma}=\left(2 \mathrm{NS} / \mathrm{C}^{\gamma}\right) \mathrm{M}_{42}^{\gamma} \mathrm{S}(3 / 2) \mathrm{m}^{10} \\
{[3.19 \mathrm{c}]} & \lambda_{44}^{\gamma}=\left(2 \mathrm{NS} / \mathrm{C}^{\gamma}\right) \mathrm{M}_{44}^{\gamma} \mathrm{S}(3 / 2) \mathrm{m}^{10} \\
{[3.19 \mathrm{~d}]} & \lambda_{21}^{\varepsilon}=\left(2 \mathrm{NS} / \mathrm{C}^{\varepsilon}\right)\left[\mathrm{M}_{21}^{\varepsilon} \mathrm{S}(1 / 2) \mathrm{m}^{3}+(2 / 3)^{\frac{1}{2}} \widetilde{\mathrm{D}}^{\varepsilon} \mathrm{Sm}^{2}\right] \\
{[3.19 \mathrm{e}]} & \lambda_{41}^{\varepsilon}=\left(2 \mathrm{NS} / \mathrm{C}^{\varepsilon}\right) \mathrm{M}_{41}^{\varepsilon} \mathrm{S}(3 / 2) \mathrm{m}^{10}
\end{array}
$$

where $\widetilde{D}^{\gamma}$ and $\widetilde{D}^{\varepsilon}$ are defined in [3.12]. These result from comparing equations [3.9] and [3.18]. The single-ion contributions again follow the $\ell(\ell+1) / 2$ power law as expected from the analysis of Kittel and Van Vleck (1960), while the two-ion terms vary as $\mathrm{m}^{2}$. The relations [3.19] enable one to express the (microscopic) 
magnetoelastic coupling constants in terms of the (macroscopic) magnetostriction constants in a fairly direct manner. For example, if the two-ion magnetoelastic effects are neglected [3.19a] and [3.19d] can be written in the form
[3.20a]
$\widetilde{B}^{\gamma}=c^{\gamma} \lambda^{\gamma} /\left[2 \mathrm{NS}\left(\mathrm{S}-\frac{1}{2}\right) \mathrm{m}^{3}\right]$
$[3.20 b]$
$\widetilde{B}^{\varepsilon}=c^{\varepsilon} \lambda^{\varepsilon} /\left[2 \mathrm{NS}\left(\mathrm{S}-\frac{1}{2}\right) \mathrm{m}^{3}\right]$

where we have used the Callen and Callen definitions [3.6] and [3.14]. (At $T=0$ [3.20a] differs from Cooper's result by a factor of (2/3), as pointed out by Brooks (1970). Note that the number of atoms in the crystal is $2 \mathrm{~N}$.)

Having found expressions for the equilibrium strains in the frozen lattice approximation and the connection between the magnetoelastic coupling constants and the magnetostriction constants, we now turn to consider the contributions that $\mathcal{H}_{\text {me }}^{I}$ and $\mathcal{H}_{\text {me }}^{I I}$ make to the stability conditions and to the spin-wave energies.

At this point it is useful to define the functions listed in Table 1, which by means of [3.15] and [3.18] can be expressed in terms of either the $\lambda_{\ell m}^{\Gamma}$ 's or Mason's $A, B, C, H$ and $I$. It can be seen that they are composed of terms having either axial or hexagonal symmetry. The temperature dependence of these functions follows from [3.19]. However, in the analysis of experimental results it may be preferable to use magnetostriction constants whose temperature dependence has been determined by experiment. 


\begin{tabular}{|c|c|c|c|}
\hline Function & Definition & $\begin{array}{l}\text { Expressed in terms } \\
\text { of the } \lambda{ }_{\ell m} \text { 's }\end{array}$ & $\begin{array}{c}\text { Expressed in terms of } \\
\text { Mason's } A, B, C, H, I \text {. }\end{array}$ \\
\hline$f^{\varepsilon}(\theta)$ & $\bar{\varepsilon}_{1}^{\varepsilon} \sin \phi+\bar{\varepsilon}_{2}^{\varepsilon} \cos \phi$ & $\lambda_{21}^{\varepsilon}\left(-\hat{Y}_{2 I}(\theta, 0)\right)+\lambda \varepsilon_{4 I}^{\varepsilon}\left(-Y_{4 I}(\theta, 0)\right)$ & $\frac{1}{2} \sin \theta \cos \theta\left(H+I \cos ^{2} \theta\right)$ \\
\hline $\mathrm{f}_{1}^{\gamma}(\theta, \phi)$ & $\bar{\varepsilon}_{1}^{\gamma} \cos 2 \phi+\bar{\varepsilon}_{2}^{\gamma} \sin 2 \phi$ & $\lambda_{22}^{\gamma} \tilde{Y}_{22}(\theta, 0)+\lambda{ }_{42}^{\gamma} \tilde{Y}_{42}(\theta, 0)$ & $\left(B \cos ^{2} \theta+C\right) \sin ^{2} \theta$ \\
\hline & & $+\cos 6 \phi \lambda \gamma_{44}^{\gamma} \Psi_{44}(\theta, 0)$ & $-\cos 6 \phi\left(\frac{1}{2} A \sin ^{4} \theta\right)$ \\
\hline $\mathrm{f}_{2}^{\gamma}(\theta, \phi)$ & $\bar{\varepsilon}_{1}^{\gamma} \cos 4 \phi-\bar{\varepsilon}_{2}^{\gamma} \sin 4 \phi$ & $\lambda_{44}^{\gamma} \tilde{Y}_{44}(\theta, 0)+\cos 6 \phi\left[\lambda_{22}^{\gamma}\right.$ & $-\frac{1}{2} A \sin ^{4} \theta+\cos 6 \phi$ \\
\hline & & $\left.x \widetilde{Y}_{22}(\theta, 0)+\lambda \gamma_{42}^{Y} \widetilde{Y}_{42}(\theta, 0)\right]$ & $x\left(B \cos ^{2} \theta+C\right) \sin ^{2} \theta$ \\
\hline$g_{1}^{\gamma}(\theta, \phi)$ & $\bar{\varepsilon}_{1}^{\gamma} \sin 2 \phi-\bar{\varepsilon}_{2}^{\gamma} \cos 2 \phi$ & $\lambda_{44}^{\gamma} \widetilde{Y}_{44}(\theta, 0) \sin 6 \phi$ & $-3 / 2 A \sin ^{4} \theta \sin 6 \phi$ \\
\hline$g_{2}^{\gamma}(\theta, \phi)$ & $\bar{\varepsilon}_{1}^{\gamma} \sin 4 \phi+\bar{\varepsilon}_{2}^{\gamma} \cos 4 \phi$ & {$\left[\lambda{ }_{22}^{\gamma} \Psi_{22}(\theta, 0)+\lambda{ }_{42}^{\gamma} \Psi_{42}(\theta, 0)\right]$} & $\left(B \cos ^{2} \theta+C\right) \sin ^{2} \theta \sin 6 \phi$ \\
\hline
\end{tabular}


Considering now the stability condition for the angle $\theta$, equation [2.19], we find that the last two lines of [2.19] must be replaced by

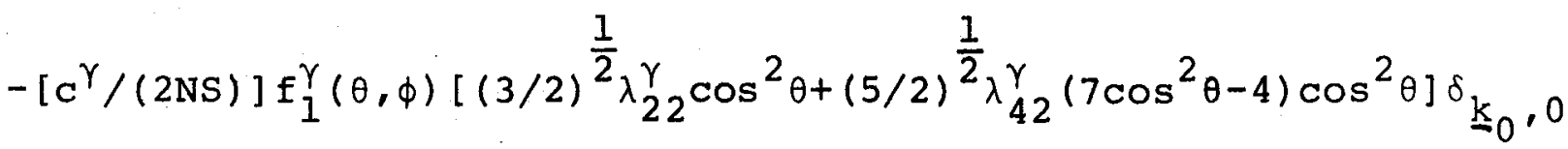

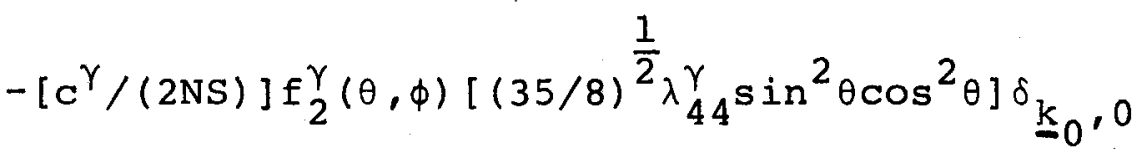

$-\left[c^{\varepsilon} /(2 N S)\right] f^{\varepsilon}(\theta)\left[(3 / 2)^{\frac{1}{2}} \lambda_{21}^{\varepsilon} \cos 2 \theta \cot \theta+(5 / 16)^{\frac{1}{2}} \lambda_{41}^{\varepsilon}\left(28 \cos ^{4} \theta-27 \cos ^{2} \theta+3\right) \cot \theta\right] \delta_{k_{0}}$ $[3.21]$

Similarly, the stability condition for $\phi$ which replaces $[2.20]$ is,

$$
\begin{gathered}
g \mu_{B}\left(H_{\xi} \sin \phi-H_{\eta} \cos \phi\right) \sin \theta m-6 B_{6}^{6} \sin ^{6} \theta \sin 6 \phi s(5 / 2) m^{21} \\
+6\left[c^{\gamma} /(2 N S)\right] g_{2}^{\gamma}(\theta, \phi) \lambda_{44}^{\gamma} \tilde{Y}_{44}(\theta, 0)=0
\end{gathered}
$$

Equations [3.18] and [3.19] have been used in arriving at these results. (We note that in the $\mathrm{T}=0$ limit with $\theta=\frac{\pi}{2}$ equation [3.22] can be shown to be equivalent to equation [3] of Nielsen et. al. $(1970) \cdot)$

Turning now to the equations of motion the commutators of the single-ion magnetoelastic terms $\mathcal{H}_{\text {me }}^{I}$ are calculated in the same way as the single-ion crystal field terms. One obtains the following contributions to $\mathrm{A}_{\underline{\underline{q}}}$ and $\mathrm{B}_{\mathrm{q}}$ (equations [2.25] and [2.26]):

$$
\begin{aligned}
A_{m e}^{I} & =f_{1}^{\gamma}(\theta, \phi)\left[3 M_{22}^{\gamma} \widetilde{Y}_{22}(\theta, 0) \mathrm{S}(1 / 2) \mathrm{m}^{2}+10 \mathrm{M}_{42}^{\gamma} \widetilde{Y}_{42}(\theta, 0) \mathrm{s}(3 / 2) \mathrm{m}^{9}\right] \\
& +f_{2}^{\gamma}(\theta, \phi)\left[10 \mathrm{M}_{44}^{\gamma} \widetilde{Y}_{44}(\theta, 0) \mathrm{s}(3 / 2) \mathrm{m}^{9}\right] \\
& +\mathrm{f}^{\varepsilon}(\theta)\left[3 \mathrm{M}_{21}^{\varepsilon}\left(-\widetilde{Y}_{21}(\theta, 0)\right) \mathrm{S}(1 / 2) \mathrm{m}^{2}+10 \mathrm{M}_{41}^{\varepsilon}\left(-\widetilde{Y}_{41}(\theta, 0)\right) \mathrm{S}(3 / 2) \mathrm{m}^{9}\right]
\end{aligned}
$$




$$
\begin{aligned}
& B_{m e}^{I}=(\sin \theta)^{-2}\left\{f _ { 1 } ^ { \gamma } ( \theta , \phi ) \left[M_{22}^{\gamma} \widetilde{Y}_{22}(\theta, 0)\left(3 \sin ^{2} \theta-4\right) \mathrm{s}(1 / 2) \mathrm{m}^{2}\right.\right. \\
& \left.+M_{42}^{Y} \tilde{Y}_{42}(\theta, 0)\left(10 \sin ^{2} \theta-4\right) \mathrm{s}(3 / 2) \mathrm{m}^{9}\right] \\
& +\mathrm{f}_{2}^{\gamma}(\theta, \phi)\left[\mathrm{M}_{44}^{\gamma} \widetilde{Y}_{44}(\theta, 0)\left(10 \sin ^{2} \theta-16\right) \mathrm{S}(3 / 2) \mathrm{m}^{9}\right] \\
& +f^{\varepsilon}(\theta)\left[M_{2 I}^{\varepsilon}\left(-\widetilde{Y}_{21}(\theta, 0)\right)\left(3 \sin ^{2} \theta-1\right) S(I / 2) m^{2}\right. \\
& \left.\left.+M_{41}^{\varepsilon}\left(-\widetilde{Y}_{4 I}(\theta, 0)\right)\left(10 \sin ^{2} \theta-1\right) S(3 / 2) m^{9}\right]\right\} \\
& +i(\cot \theta / \sin \theta)\left\{g _ { 1 } ^ { \gamma } ( \theta , \phi ) \left[2 M_{22}^{\gamma} \widetilde{Y}_{22}(\theta, 0) s(1 / 2) \mathrm{m}^{2}\right.\right. \\
& \left.+6 M_{42}^{\gamma} \widetilde{Y}_{42}(\theta, 0) \mathrm{S}(3 / 2) \mathrm{m}^{9}-4(15)^{\frac{1}{2}} \mathrm{M}_{42}^{\gamma} \widetilde{\mathrm{Y}}_{22}(\theta, 0) \mathrm{S}(3 / 2) \mathrm{m}^{9}\right] \\
& \left.+g_{2}^{\gamma}(\theta, \phi)\left[12 M_{44}^{\gamma} \widetilde{Y}_{44}(\theta, 0) S(3 / 2) \mathrm{m}^{9}\right]\right\}
\end{aligned}
$$

The two-ion magnetoelastic terms $\mathcal{H}_{\text {me }}$ are handled in the same manner as the exchange terms. Their contributions to ${ }^{A} \underline{q}^{\prime}{ }^{B}{ }^{\prime}$ ' $\mathrm{C}_{\underline{q}}$ and $\mathrm{D}_{\underline{\mathrm{q}}}$ (equations $[2.25]-[2.28]$ ) are,

$$
\mathrm{A}_{\mathrm{me}}^{\mathrm{II}}(\underline{q})=\mathrm{f}_{1}^{\gamma}(\theta, \phi)\left[\frac{1}{4} \widetilde{D}^{\gamma}(\underline{q})+\tilde{D}^{\gamma}\right] \sin ^{2} \theta \mathrm{Sm}+\mathrm{f}^{\varepsilon}(\theta)\left[\frac{1}{4} \tilde{D}^{\varepsilon}(\underline{q})+\tilde{D}^{\varepsilon}\right] \sin 2 \theta \mathrm{Sm}
$$

[3.24a]

[3.24b]

$$
\begin{aligned}
B_{m e}^{I I}(\underline{q}) & =f^{\varepsilon}(\theta)\left[\frac{1}{4} \widetilde{D}^{\varepsilon}(q) \sin 2 \theta-\widetilde{D}^{\varepsilon} \cos 2 \theta \cot \theta\right] \mathrm{Sm} \\
& -f_{1}^{\gamma}(\theta, \phi)\left[\frac{1}{4} \widetilde{D}^{\gamma}(\underline{q})\left(1+\cos ^{2} \theta\right)+\widetilde{D}^{\gamma} \cos ^{2} \theta\right] \mathrm{Sm} \\
& -i g_{1}^{\gamma}(\theta, \phi)\left[\frac{1}{2} \widetilde{D}^{\gamma}(\underline{q}) \cos \theta\right] \mathrm{Sm}
\end{aligned}
$$

$$
C_{m e}^{I I}(\underline{q})=f_{1}(\theta, \phi)\left[\frac{1}{4} \widetilde{D}^{\gamma^{\prime}}(\underline{q}) \sin ^{2} \theta\right] \mathrm{Sm}+\mathrm{f}^{\varepsilon}(\theta)\left[\frac{\mathrm{l}}{4} \widetilde{D}^{\varepsilon^{\prime}}(\underline{q}) \sin 2 \theta\right] \mathrm{Sm}
$$




$$
D_{m e}^{I I}(\underline{q})=-f_{1}^{\gamma}(\theta, \phi)\left[\frac{1}{4} \widetilde{D}^{\prime}(\underline{q})\left(1+\cos ^{2} \theta\right)\right] \mathrm{Sm}+f^{\varepsilon}(\theta)\left[\frac{1}{4} \tilde{D}^{\varepsilon^{\prime}}(\underline{q}) \sin 2 \theta\right] \mathrm{Sm}
$$

$$
-i g_{1}^{\gamma}(\theta, \phi)\left[\frac{1}{2} D^{\gamma^{\prime}}(q) \cos \theta\right] \mathrm{Sm}
$$

where $\tilde{D}^{\gamma}$ and $\widetilde{D}^{\varepsilon}$ are defined in [3.12], and $f^{\varepsilon}(\theta), f_{1}^{\gamma}(\theta, \phi)$ and $g_{1}^{\gamma}(\theta, \phi)$ are given in Table 1 .

Finally, for use in the next section, we form the combinations which enter the two brackets of the expression for the spinwave energy $\mathrm{E}_{\underline{q}}^{(i)}$, equation $[2.40]$, in the special case that $\mathrm{B}_{\underline{q}}$ and $\mathrm{D}_{\mathrm{q}}$ are real:

[3.25a] $A_{m e}^{I}+B_{m e}^{I}+A_{m e}^{I I}(\underline{q})+B_{m e}^{I I}(\underline{q}) \pm\left[C_{m e}^{I I}(\underline{q})+D_{m e}^{I I}(\underline{q})\right]$

$$
=M_{m e}(\theta, \phi)-P_{m e}^{(i)}(\underline{q}) f^{\varepsilon}(\theta) \sin \theta \cos \theta+\frac{1}{2} Q m e(\underline{i}) f_{1}^{\gamma}(\theta, \phi) \cos ^{2} \theta
$$

$[3.25 b]$

$$
\begin{gathered}
A_{m e}^{I}-B_{m e}^{I}+A_{m e}^{I I}(\underline{q})-B_{m e}^{I I}(\underline{q}) \pm\left[C_{m e}^{I I}(\underline{q})-D_{m e}^{I I}(\underline{q})\right] \\
=N_{m e}(\theta, \phi)-\frac{1}{2} Q_{m e}^{(i)}(\underline{q}) E_{1}^{\gamma}(\theta, \phi)
\end{gathered}
$$

where,

$$
\begin{aligned}
M_{\mathrm{me}}(\theta, \phi)=\left[c^{\varepsilon} /\left(2 \mathrm{NSmsin}{ }^{2} \theta\right)\right] \mathrm{f}^{\varepsilon}(\theta)\left[\lambda_{21}^{\varepsilon} \widetilde{Y}_{21}(\theta, 0)\left(1-6 \sin ^{2} \theta\right)\right. & \\
& \left.+\lambda_{41}^{\varepsilon} \widetilde{Y}_{41}(\theta, 0)\left(1-20 \sin ^{2} \theta\right)\right] \\
+ & {\left.\left[c^{\gamma /(2 N S m s i n}{ }^{2} \theta\right)\right] \mathrm{f}_{1}^{\gamma}(\theta, \phi)\left[\lambda_{22}^{\gamma} \widetilde{\mathrm{Y}}_{22}(\theta, 0)\left(6 \sin ^{2} \theta-4\right)\right.} \\
& \left.+\lambda_{42}^{\gamma} \widetilde{Y}_{42}(\theta, 0)\left(20 \sin ^{2} \theta-4\right)\right]
\end{aligned}
$$

$[3.26 a]$

$$
+\left[c^{\gamma} /\left(2 N S m \sin ^{2} \theta\right)\right] f_{2}^{\gamma}(\theta, \phi)\left[\lambda_{44}^{\gamma} \widetilde{Y}_{44}(\theta, 0)\left(20 \sin ^{2} \theta-16\right)\right]
$$




$$
\begin{aligned}
& \mathrm{N}_{\mathrm{me}}(\theta, \phi)=\left[\mathrm{c}^{\varepsilon} /\left(2 \mathrm{NSmsin} \sin ^{2} \theta\right)\right]\left[f^{\varepsilon}(\theta)\right]^{2} \\
& +\left[c^{\gamma} /\left(2 N \operatorname{Smsin}^{2} \theta\right)\right] \mathrm{f}_{1}^{\gamma}(\theta, \phi)\left[4 \lambda_{22}^{\gamma} \widetilde{\mathrm{Y}}_{22}(\theta, 0)+4 \lambda_{42}^{\gamma} \widetilde{\mathrm{Y}}_{42}(\theta, 0)\right] \\
& {[3.26 \mathrm{~b}]+\left[\mathrm{c}^{\gamma} /\left(2 \mathrm{NSmsin}{ }^{2} \theta\right)\right] \mathrm{f}_{2}^{\gamma}(\theta, \phi)\left[16 \lambda_{44}^{\gamma} \widetilde{Y}_{44}(\theta, 0)\right]} \\
& \text { [3.27a] } \\
& P_{m e}^{(i)}(q)=\operatorname{Sm}\left[2 \tilde{D}^{\varepsilon}-\tilde{D}^{\varepsilon}(q) \pm \tilde{D}^{\varepsilon^{\prime}}(q)\right] \\
& \text { [3.27b] } \\
& Q_{m e}^{(i)}(\underline{q})=\operatorname{Sm}\left(2 \tilde{D}^{\gamma}-\widetilde{D}^{\gamma}(\underline{q}) \pm \widetilde{D}^{\gamma^{\prime}}(q)\right]
\end{aligned}
$$

The various symbols occurring in these equations are defined in [3.10]-[3.13] and in Table 1. Regarding the \pm signs in [3.25] and [3.27] we note that the plus sign taken throughout pertains to the upper spin-wave mode (labelled $i=2$ ) while the minus sign throughout pertains to the lower spin-wave mode (labelled $i=1$ ).

\subsection{The Mobile Lattice Approximation}

If the strains associated with magnetostriction can follow the nearly uniform magnetization in the long wave-length spinwave modes one has the other limiting approximation, which for simplicity we denote the "mobile lattice approximation". Cooper (1968a) has discussed this approximation from a macroscopic point of view. For a magnetoelastic coupling linear in $\varepsilon_{1}^{\gamma}$ and $\varepsilon_{2}^{\gamma}$ and up to fourth power in the direction cosines of the (uniform) magnetization he found that the resulting Hamiltonian consists of two parts, one axially symmetric about the hexagonal axis, the other having hexagonal symmetry about this axis. These terms will 
then affect the spin-wave energies in a manner similar to that of the crystal field terms of corresponding symmetry.

As an aid to describing the mobile lattice approximation in the present formulation of the magnetostrictive terms we introduce local strain functions $\varepsilon^{\Gamma}\left(\underline{S}_{i}\right)$ each of which depends on the instantaneous direction of $\underline{S}_{i}$. The direction of $\underline{S}_{i}$ is given by angles $\left(\theta_{i}, \phi_{i}\right)$ with respect to the crystal axes while $(\theta, \phi)$ wili as usual specify the direction of the static magnetization. Requiring. that the local strains depend on $\underline{s}_{i}$ implies that the strains closely follow the motion of the spins. Of course, this is consistent with the original assumption of uniform strains only for the $q=0$ spin-wave mode in which the spins $\underline{S}_{i}$ precess in phase. However it is precisely for this case that the mobile lattice approximation appears to be useful (Cooper 1968a, Brooks 1970). Let us confine our attention to the terms in $\mathcal{R}_{e}$ and $\mathcal{K}_{\mathrm{me}}$ belonging to the irreducible representation $\Gamma_{\gamma}$. Then the magnetostrictive terms in the Hamiltonian may be written as

$$
\mathcal{H e}_{e}=\left[\mathrm{c}^{\gamma} /(2 \mathrm{~N})\right] \underset{i}{\sum}\left\{\frac{1}{2}\left[\varepsilon_{1}^{\gamma}\left(\underline{S}_{i}\right)\right]^{2}+\frac{1}{2}\left[\varepsilon_{2}^{\gamma}\left(\underline{S}_{i}\right)\right]^{2}\right\}
$$

[3.29]

$$
\begin{aligned}
& \mathcal{H}_{\text {me }}^{I}=-M_{22}^{\gamma} \sum_{i}\left[\varepsilon_{1}^{\gamma}\left(\underline{s}_{i}\right) \widetilde{\sigma}_{22}^{+}\left(\underline{s}_{i}\right)+\varepsilon_{2}^{\gamma}\left(\underline{s}_{i}\right) \widetilde{\sigma}_{22}^{-}\left(\underline{s}_{i}\right)\right] \\
& -M_{42}^{\gamma} \sum_{i}\left[\varepsilon_{1}^{\gamma}\left(\underline{s}_{i}\right) \widetilde{O}_{42}^{+}\left(\underline{s}_{i}\right)+\varepsilon_{2}^{\gamma}\left(\underline{s}_{i}\right) \widetilde{O}_{42}^{-}\left(\underline{s}_{i}\right)\right] \\
& -M_{44}^{\gamma} \sum_{i}\left[\varepsilon_{1}^{\gamma}\left(\underline{S}_{i}\right) \widetilde{O}_{44}^{+}\left(\underline{S}_{i}\right)-\varepsilon_{2}^{\gamma}\left(\underline{S}_{i}\right) \tilde{O}_{44}^{-}\left(\underline{S}_{i}\right)\right]
\end{aligned}
$$


Minimizing $\mathcal{H}_{e}+\mathcal{H}_{\text {me }}^{I}$ with respect to the local strains one obtains

$$
\begin{aligned}
& \text { [3.30a] } \varepsilon_{1}^{\gamma}\left(\underline{S}_{i}\right)=\left(2 \mathrm{~N} / \mathrm{c}^{\gamma}\right)\left[\mathrm{M}_{22}^{\gamma} \widetilde{\mathrm{O}}_{22}^{+}\left(\underline{S}_{i}\right)+\mathrm{M}_{42}^{\gamma} \widetilde{\mathrm{O}}_{42}^{+}\left(\underline{S}_{i}\right)+\mathrm{M}_{44}^{\gamma} \widetilde{\mathrm{O}}_{44}^{+}\left(\underline{S}_{i}\right)\right] \\
& {[3.30 \mathrm{~b}] \quad \varepsilon_{2}^{\gamma}\left(\underline{S}_{i}\right)=\left(2 \mathrm{~N} / \mathrm{c}^{\gamma}\right)\left[\mathrm{M}_{22}^{\gamma} \widetilde{\mathrm{O}}_{22}^{-}\left(\underline{S}_{i}\right)+M_{42}^{\gamma} \widetilde{\mathrm{O}}_{42}^{-}\left(\underline{S}_{i}\right)-M_{44}^{\gamma} \widetilde{\mathrm{O}}_{44}^{-}\left(\underline{S}_{i}\right)\right] .}
\end{aligned}
$$

We note in passing that the frozen lattice approximation can be obtained directly from these expressions by taking a time average (or, equivalently, a thermal average) at each site, with the result that the equilibrium strains depend only on the direction of the magnetization expressed by $(\theta, \phi)$.

On substituting equation [3.30] for the local strains back into $\mathcal{H}_{e}$ and $\mathcal{H}_{\text {me }}^{I}$ one obtains for the resulting Hamiltonian sums of terms like,

[3.31] $\left[\tilde{o}_{22}^{+}\left(\underline{S}_{i}\right)\right]^{2}, \quad \tilde{o}_{22}^{+}\left(\underline{s}_{i}\right) \tilde{o}_{42}^{+}\left(\underline{S}_{i}\right), \tilde{o}_{22}^{+}\left(\underline{S}_{i}\right) \tilde{o}_{44}^{+}\left(\underline{S}_{i}\right)$, etc.

which transform under rotations of the vector $\underline{S}_{i}$ in the same way as [3. 32] $\left[\tilde{Y}_{22}^{+}\left(\theta_{i}, \phi_{i}\right)\right]^{2}, \widetilde{Y}_{22}^{+}\left(\theta_{i} \phi_{i}\right) \widetilde{Y}_{42}^{+}\left(\theta_{i}, \phi_{i}\right), \widetilde{Y}_{22}^{+}\left(\theta_{i} \phi_{i}\right) \widetilde{Y}_{44}^{+}\left(\theta_{i} \phi_{i}\right)$, etc.

Such combinations can then be decomposed using the spherical harmonic addition theorem. One finds that the resulting terms have either axial symmetry or hexagonal symmetry, exactly as in Cooper's calculation. Of course these terms can be put back into operator equivalents and it is clear that they then look like the 
axial and hexagonal crystal field terms. For example, the magnetostrictive terms make an effective contribution to $B_{6}^{6}$ of

$$
-\left(2 N / C^{\gamma}\right)\left[\frac{(105)^{\frac{1}{2}}}{32} M_{22}^{\gamma} M_{44}^{\gamma}-\frac{(7)^{\frac{1}{2}}}{24} M_{42}^{\gamma}{ }_{44}^{\gamma}\right]
$$

We note that this is a temperature independent constant. If it is desired to express it in terms of measurable quantities the relations [3.19] at $\mathrm{T}=0$ can be used, giving,

[3.34] $-\left[c^{\gamma} /(2 N)\right]\left[\frac{(105)^{\frac{1}{2}} \lambda_{22}^{\gamma}(0) \lambda_{44}^{\gamma}(0)}{32 S^{2} s(1 / 2) S(3 / 2)}-\frac{(7)^{\frac{1}{2}} \lambda_{42}^{\gamma}(0) \lambda_{44}^{\gamma}(0)}{24 S^{2} s(3 / 2) s(3 / 2)}\right]$

At this point our work differs in philosophy from that of Cooper in that we do not relate the "dynamic strains" of equation [3.30] to the saturation magnetostriction constants through a relation of the type [3.16]. In our view the magnetostriction constants, which depend on temperature, are related to the equilibrium strains of the last subsection obtained from thermal averages of the spin operators. Consequently our expression above contains factors $S^{2} S(1 / 2) S(3 / 2)$ and $S^{2} S(3 / 2) S(3 / 2)$ in the denominators arising from the thermal averages. Cooper introduced temperature dependence into his expression for the spin-wave energy by allowing the magnetostriction constants to vary with temperature according to the Callen and Callen theory. The magnetoelastic contribution to $\mathrm{B}_{6}^{6}$ in his theory is, in our notation,

$[3.35]$

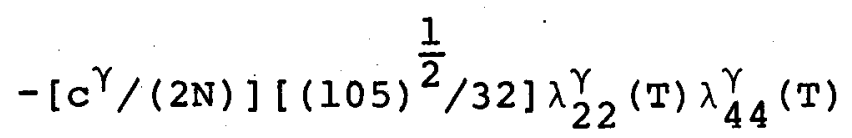


which varies as $\mathrm{m}^{13}$ at low temperatures. (Note that Cooper did not include a magnetoelastic term corresponding to $M_{42}^{\gamma}$ in his calculation.) In our work, however, the magnetoelastic contribution to $\mathrm{B}_{6}^{6}$ in the mobile lattice approximation is temperature independent, but, like the crystal field part of $B_{6}^{6}$, enters the expression for the spin-wave energy with a dependence going as $\mathrm{m}^{21}$.

We conclude that the mobile lattice approximation yields for the lower $q=0$ spin-wave energy in the case of a planar ferromagnet a result of the form,

$$
\hbar_{\omega}(0)=(L)^{\frac{1}{2}}\left(g \mu_{B} H-36 B_{6}^{6} \cos 6 \phi m^{21}\right)^{\frac{1}{2}}
$$

where I stands for a sum of large axial crystal fields and magnetoelastic terms, and $B_{6}^{6}$ contains a temperature independent contribution [3.33] or [3.34]. (It has been assumed that the direction of the magnetization coincides with that of the applied field.) Thus by applying a field along a hard direction ( $\phi=0$ if ${ }^{6} 6$ is positive; $\phi=\pi / 6$ if $B_{6}^{6}$ is negative) the resonance frequency can be reduced to zero. The same is true in Cooper's theory but the temperature dependence of the magnetoelastic contribution is different. For magnetic resonance at frequencies below about $40 \mathrm{GHz}$ the corresponding value of $\hbar \omega(0)$ is considerably below the gap which occurs in the frozen lattice approximation. For such cases the mobile lattice approximation provides a plausible explanation of the experimental results (Cooper 1968a, Brooks 1970). 


\section{CHAPTER 4}

\section{ANALYSIS OF PARTICULAR SPIN STRUCTURES}

In this section we shall discuss separately the different spin structures to which the theory we have presented is applicable. The relevant equations of the preceding sections are the conditions for stable equilibrium, equations [2.19] and [2.20]; equations $[2.25]-[2.28]$ and [3.24]-[3.27] for coefficients of the linearized equations of motion; and the solutions to the eigenvalue problem, equations $[2.37]-[2.40]$ in appropriate circumstances. The expressions which we give for the spin-wave energies in different cases will be used in subsequent work to analyze the results of inelastic neutron scattering and magnetic resonance experiments. Here we examine only briefly the form of the contributions from different sources such as anisotropic exchange, applied field and magnetoelastic effects.

\subsection{The Planar Ferromagnet}

The low temperature phases of $\mathrm{Tb}$ and $\mathrm{Dy}$ are ferromagnetic with the spins lying in the hexagonal plane. The easy axis of magnetization is the b-direction in $\mathrm{Tb}(\phi=\pi / 6)$ and the a-direction in Dy $(\phi=0)$. Equations [2.25]-[2.28] and [3.24]-[3.27] simplify on putting $\mathrm{k}_{0}=0$ and $\phi=\pi / 2$. In particular $\mathrm{B}_{\mathrm{q}}$ is real, and

$$
\begin{aligned}
& \text { [4. 1] } \quad \mathrm{C}_{\underline{q}}=-S\left[J^{\prime}(\underline{q})+\frac{1}{2} K^{\prime}(\underline{q})+\left(\frac{1}{4}\right)\left(\frac{1}{2} A \cos 6 \phi-C\right) \tilde{D}^{\prime} \cdot(\underline{q})\right] \mathrm{m} \\
& {[4.2] \quad \mathrm{D}_{\underline{q}}=-S\left[\frac{1}{2} K^{\prime}(\underline{q})-\left(\frac{1}{4}\right)\left(\frac{1}{2} A \cos 6 \phi-C\right) \tilde{D}^{\prime}(q)\right] \mathrm{m}}
\end{aligned}
$$

where A and C are Mason's temperature-dependent magnetostriction 
constants, equations $[3.15 \mathrm{a}]$ and $[3.15 \mathrm{c}]$, and $\widetilde{D}^{\gamma}(\underline{q})$ is defined in [3.11b]. In general $\mathrm{C}_{\mathrm{q}}$ and $\mathrm{D}_{\mathrm{q}}$ are complex and therefore the expressions [2.38]-[2.39] must be used for the spin-wave energies. However, when $q$ lies in the plane ГKHA or the plane KMLH of Fig. 1 , equation [2.40] can be used. With the help of the stability condition [2.19] we obtain in this case,

$$
\begin{aligned}
& {[4.3] \underset{\underline{q}}{E^{(i)}}=\left\{S\left[J(0)-(J(q)+K(q))+J^{\prime}(0) \pm\left(J^{\prime}(q)+K^{\prime}(q)\right)\right] m-2 \tilde{B}(\pi / 2)\right.} \\
& \left.-6{ }_{6}^{6} S\left(\frac{5}{2}\right) \cos 6 \phi m^{20}+g \mu_{B}\left(H_{a} \cos \phi+H_{b} \sin \phi\right)+M_{m e}(\pi / 2, \phi)\right\}^{\frac{3}{2}} \\
& x \quad s\left[J(0)-J(q)+J^{\prime}(0) \pm J^{\prime}(q)\right] m-36 B_{6}^{6} S\left(\frac{5}{2}\right) \cos 6 \phi m^{20} \\
& \left.+g \mu_{B}\left(H_{a} \cos \phi+H_{b} \sin \phi\right)+N_{m e}(\pi / 2, \phi)+\frac{1}{2}\left(\frac{1}{2} A \cos 6 \phi-C\right) Q_{m e}^{(i)}(q)\right\}^{\frac{1}{2}}
\end{aligned}
$$

where $Q_{m e}^{(i)}(q)$ is defined in $[3.27 b]$ and

$$
\begin{aligned}
& \text { [4.4] } \widetilde{\mathrm{B}}(\pi / 2)=3\left(-\frac{1}{2}\right) \widetilde{\mathrm{B}}_{2}^{0} \mathrm{~S}\left(\frac{1}{2}\right) \mathrm{m}^{2}+10\left(\frac{3}{8}\right) \tilde{\mathrm{B}}_{4}^{0} \mathrm{~S}\left(\frac{3}{2}\right) \mathrm{m}^{9}+21\left(-\frac{5}{16}\right) \widetilde{\mathrm{B}}_{6}^{0} \mathrm{~S}\left(\frac{5}{2}\right) \mathrm{m}^{20} \\
& \text { [4.5] } M_{m e}(\pi / 2, \phi)=\left[C^{\gamma /(2 N S m)}\right]\left[A^{2}+2 C^{2}-2 B C+A(B-3 C) \cos 6 \phi\right] \\
& \text { [4.6] } \quad \mathrm{N}_{\mathrm{me}}(\pi / 2, \phi)=\left[\mathrm{C}^{\gamma /(2 \mathrm{NSm})}\right]\left[4\left(\mathrm{~A}^{2}+\mathrm{C}^{2}\right)-10 \mathrm{AC \operatorname {cos }} 6 \phi\right] \text {. }
\end{aligned}
$$

Here $A, B$ and $C$ are Mason's temperature-dependent magnetostriction constants (equation [3.15]). Knowing these and the elastic constant $c^{\gamma}$, as well as the reduced magnetization $m$, one can readily calculate $M_{m e}(\pi / 2, \phi)$ and $N_{m e}(\pi / 2, \phi)$. These magnetoelastic contributions to $\mathrm{E}_{\mathrm{q}}^{(i)}$ are exactly the same as those given by wielsen et al (1970) 
The two-ion magnetoelastic contribution involving $Q_{m e}^{(i)}(q)$, which occurs in the same bracket as the hexagonal crystal field, is a new result. It has exactly the same form as the isotropic exchange terms but is multiplied by the temperature-dependent factor $\left(\frac{1}{2} A \cos 6 \phi-C\right)$. The fact that this term enters only the second bracket of [4.3] while the anisotropic exchange terms $K(q)$ and $K^{\prime}(q)$ enter only the first bracket has a simple interpretation. Terms in the first bracket other than from isotropic exchange and applied field represent an effective field along the hexagonal axis, while terms in the second bracket other than from isotropic exchange and applied field represent an effective field in the hexagonal plane. Viewed semiclassically, it is these two effective fields perpendicular to the magnetization which are experienced by the spins in precessing at the increased amplitude of the spin-wave excitation. Note, however, that the applied field, which was assumed to lie in the hexagonal plane (since a component $\mathrm{H}_{\mathrm{C}}$ is inconsistent with the assumption $\theta=\frac{\pi}{2}$ ), enters both brackets in the same way, a result consistent with elementary spin-wave theory for a Hamiltonian consisting only of isotropic exchange and applied magnetic field. (Note also that an amount $-6 \mathrm{~B}_{6}^{6} \mathrm{~S}\left(\frac{5}{2}\right)$ $\cos 6 \phi \mathrm{m}^{20}$ from the hexagonal crystal field enters both brackets, acting in this respect like an applied field.) Finally, it is worth pointing out that if the direction of magnetization coincides with the direction of $\underline{H}$ the magnetic field terms in [4.3] reduce to $g \mu_{B} \mathrm{H}$ with $\mathrm{H}$ the magnitude of the field. The crystal field contributions to [4.3] are usually 
estimated from measurements of the anisotropy coefficients. In making such estimates, however, it is necessary to exclude magnetostrictive contributions to the anisotropy coefficients, a task which is difficult to carry out in practice. We shall not discuss this further in this paper.

It remains to be mentioned that the \pm signs in [4.3] give the two spin-wave modes which we label by $i=1,2$. It can be shown that $J^{\prime}(q), \tilde{D}^{\gamma^{\prime}}(q), \tilde{D}^{\varepsilon^{\prime}}(\underline{q})$, and $K^{\prime}(\underline{q})$ vanish at all points in the plane AHL and along the line $\mathrm{KH}$ of $\mathrm{Fig.} \mathrm{1.} \mathrm{As} \mathrm{a} \mathrm{result} \mathrm{the} \mathrm{modes} \mathrm{are} \mathrm{degenerate}$ at these points.

of particular interest are the frequencies for magnetic resonance which follow from [4.3] putting $q=0$. These are

[4.7a] $h \nu_{1}=\left\{g \mu_{B} H+g \mu_{B}\left(N^{x}-N^{z}\right) M_{0} m-S\left(K(0)+K^{\prime}(0)\right) m\right.$

$$
\begin{aligned}
& \left.-2 \tilde{B}(\pi / 2)-6 B \frac{6}{6} S\left(\frac{5}{2}\right) \cos 6 \phi m^{20}+M_{m e}(\pi / 2, \phi)\right\}^{\frac{1}{2}} \\
& x\left\{g \mu_{B} H+g \mu_{B}\left(N^{Y}-N^{2}\right) M_{0} m-36 B \frac{6}{6} S\left(\frac{5}{2}\right) \cos 6 \phi m^{20}+N_{m e}(\pi / 2, \phi)\right\}^{\frac{1}{2}}
\end{aligned}
$$

$[4.7 b] \quad h \nu_{2}=\left\{g \mu_{B} H+g \mu_{B}\left(N^{X}-N^{z}\right) M_{0} m+S\left[2 J^{\prime}(0)-K(0)+K^{\prime}(0)\right] m\right.$

$$
\begin{aligned}
& \left.-2 \widetilde{B}(\pi / 2)-6 B_{6}^{6} S\left(\frac{5}{2}\right) \cos 6 \phi m^{20}+M_{m e}(\pi / 2, \phi)\right\}^{\frac{1}{2}} \\
& x\left\{g \mu_{B} H+g \mu_{B}\left(N^{Y}-N^{2}\right) M_{0} m+2 S J^{\prime}(0)-36 B \frac{6}{6} S\left(\frac{5}{2}\right) \cos 6 \phi m^{20}\right. \\
& \left.+N_{m e}(\pi / 2, \phi)+\left(\frac{1}{2} A \cos 6 \phi-C\right) S \tilde{S D}^{\gamma}(0) m\right\}^{\frac{1}{2}}
\end{aligned}
$$

where $v_{1}$ is the lower frequency and $v_{2}$ the higher frequency. Here 
$\mathrm{M}_{0}$ is the saturation magnetization at $\mathrm{T}=0 \quad\left(\mathrm{M}_{0}=2 \mathrm{Ng} \mu_{B} \mathrm{~S}\right)$. We have introduced the demagnetizing fields (Kittel 1948, Keffer 1966, Cooper et. al. 1962, Brooks et. al.1968) whose effect is relatively more important for the resonance frequencies than for spin-waves away from $q=0 . \quad N^{x}, N^{Y}$ and $N^{z}$ are the usual demagnetizing factors $\left(N^{x}+N^{Y}+N^{z}=4 \pi\right), N^{z}$. referring to the direction of magnetization, $N^{x}$ to the direction along the hexagonal axis and $N^{Y}$ to a mutually perpendicular direction lying in the hexagonal plane.

\subsection{The Nonplanar Ferromagnet}

This structure, in which the magnetization makes an angle $\theta \neq \pi / 2$ with the hexagonal axis, occurs in $\mathrm{Tb}$ and $D \mathrm{y}$ when a magnetic field is applied along the c-axis, and there is evidence (Jordan and Lee 1967) that even in zero applied field Dy has this structure below 90K. Also the fan structure, which appears in the low temperature phases of Ho and Er for a moderately large field in the hexagonal plane, will approach a pure ferromagnet at higher field strengths. In each case the angle $\theta$ will be given by the stability condition [2.19] with $\underline{k}_{0}=0$.

For general q's and arbitrary $\phi$ the spin-wave energies must be determined from equations [2.38]-[2.39], as in the case of the planar ferromagnet. However, if $\phi=0$ and if $q$ is in the plane $\mathrm{TKHA}$ or $\mathrm{KMLH}$ of $\mathrm{Fig} .1$, then $\mathrm{C}_{\mathrm{q}}$ and $\mathrm{D}_{\mathrm{q}}$ are real and equation [2.40] can be used. The condition $\phi=0$ also makes $B_{q}$ real. Then under these conditions equation [2.40] gives

$$
\text { [4.8] } \mathrm{E}_{\underline{q}}^{(i)}=\left\{S \left[J(0)+2 \cos ^{2} \theta K(0)-\left(J(\underline{q})+\sin ^{2} \theta K(\underline{q})\right)+J^{\prime}(0)+2 \cos ^{2} \theta K^{\prime}(0)\right.\right.
$$




$$
\begin{aligned}
& \left. \pm\left(J^{\prime}(\underline{q})+\sin ^{2} \theta K^{\prime}(q)\right)\right] m-2 B^{2}(\theta)-6 B_{6}^{6} S\left(\frac{5}{2}\right) \sin ^{6} \theta\left(1-6 \cot ^{2} \theta\right) m^{20} \\
& +2 g \mu_{B} H_{C} \cos \theta+g \mu_{B} H_{a}\left(\sin ^{2} \theta-\cos ^{2} \theta\right) / \sin \theta+M_{m e}(\theta, 0) \\
& \left.-\dot{f}^{\varepsilon}(\theta) \sin \theta \cos \theta P_{m e}^{(i)}(\underline{q})+\frac{1}{2} F_{1}^{\gamma}(\theta, 0) \cos ^{2} \theta Q_{m e}^{(i)}(\underline{q})\right\}^{\frac{1}{2}} \\
& x\left\{S\left[J(0)-J(\underline{q})+J^{\prime}(0) \pm J^{\prime}(\underline{q})\right] m-36 B_{6}^{6} S\left(\frac{5}{2}\right) \sin ^{4} \theta\right. \\
& \left.+g \mu_{B} H_{a} / \sin \theta+N_{m e}(\theta, 0)-\frac{1}{2} f_{1}^{\gamma}(\theta, 0) Q_{m e}^{(i)}(\underline{q})\right\}^{\frac{1}{2}}
\end{aligned}
$$

where

$$
\begin{aligned}
M_{m e}(\theta, 0) & =\left[C^{\varepsilon} \cos ^{2} \theta /(8 N S m)\right]\left(H+I \cos ^{2} \theta\right)\left[H\left(6 \sin ^{2} \theta-1\right)\right. \\
& \left.+I\left(5 \cos ^{2} \theta+20 \sin ^{2} \theta \cos ^{2} \theta-6\right)\right] \\
& +\left[C^{\gamma} \sin ^{2} \theta /(8 N S m)\right]\left[2\left(C+B \cos ^{2} \theta\right)-A \sin ^{2} \theta\right]\left[2 C\left(6 \sin ^{2} \theta-4\right)\right. \\
& \left.+4 B\left(\sin ^{2} \theta+10 \sin ^{2} \theta \cos ^{2} \theta-2\right)-4 A \sin ^{2} \theta\left(5 \sin ^{2} \theta-4\right)\right]
\end{aligned}
$$

[4.10]

$$
\begin{aligned}
N_{m e}(\theta, 0) & =\left[c^{\varepsilon} \cos ^{2} \theta /(8 N S m)\right]\left(H+I \cos ^{2} \theta\right)^{2} \\
& +\left[c^{\gamma} \sin ^{2} \theta /(N S m)\right]\left[2\left(C+B \cos ^{2} \theta\right)-A \sin ^{2} \theta\right]\left[\left(C+B \cos ^{2} \theta\right)-2 A \sin ^{2} \theta\right]
\end{aligned}
$$

$\tilde{B}(\theta)$ is defined in $[2.29]$ and $f^{\varepsilon}(\theta), f^{\gamma}(\theta, 0)$ are given in Table 1 . The applied field has been assumed to have components $\mathrm{H}_{\mathrm{a}}$ and $\mathrm{H}_{\mathrm{C}}$ only, consistent with the assumption $\phi=0$. Of course most of the remarks following equation [4.6] in the last subsection are applicable to equation [4.8] as well. We note that in the present 
case the modes are degenerate at all points in the plane AHL and along the line $\mathrm{KH}$ of Fig. 1.

\subsection{The Planar Spiral}

This type of structure occurs in $\mathrm{Tb}$ in the temperature range $220-228 \mathrm{~K}$, in Dy in the temperature range $85-179 \mathrm{~K}$, and in

Ho in the temperature range $20-133 \mathrm{~K}$. In equations [2.25]-[2.28] we put $\theta=\pi / 2$ and omit the terms involving the hexagonal anisotropy (for the reason mentioned in section 2.5 ) and the magnetic field (since a component $\mathrm{H}_{\mathrm{C}}$ is inconsistent with the assumption $\theta=\pi / 2$, and the effect of components $H_{a}$ and $H_{b}$ averages to zerol. We also omit the magnetoelastic terms since homogeneous strains do not exist in a spiral spin configuration.

As in the preceding cases $\mathrm{C}_{\mathrm{g}}$ and $\mathrm{D}_{\mathrm{q}}$ are complex in general, requiring the use of equations [2.38] $-[2.39]$. However, when $q$ lies in the plane TKHA or the plane KMLH, equation [2.40] may be used, giving,

$$
\begin{aligned}
& {[4.11] \mathrm{E}_{\underline{q}}^{(i)}=\left\{S\left[J\left(\underline{k}_{0}\right)-(J(q)+K(\underline{q}))+J^{\prime}\left(\underline{k}_{0}\right) \pm\left(J^{\prime}(\underline{q})+K^{\prime}(\underline{q})\right)\right] m-2 \widetilde{B}(\pi / 2)\right\}^{\frac{1}{2}}} \\
& x\left\{S \left[J\left(\underline{k}_{0}\right)-\frac{1}{2}\left(J\left(\underline{q}+\underline{k}_{0}\right)+J\left(\underline{q}-\underline{k}_{0}\right)\right)+J^{\prime}\left(\underline{k}_{0}\right) \pm \frac{1}{2}\left(J^{\prime}\left(q \underline{q}+\underline{k}_{0}\right)\right.\right.\right. \\
& \left.\left.\left.+J^{\prime}\left(\underline{q}-\underline{k}_{0}\right)\right)\right] m\right\}^{\frac{1}{2}}
\end{aligned}
$$

where $\tilde{B}(\pi / 2)$ is given in [4.4]. It may easily be seen that the lower spin-wave branch is zero at $q=0$ as must be the case for a spiral structure (Elliott and Lange 1966). The energy of the upper spin-wave branch at $q=0$ is 
$[4.12] h \nu_{2}=\left\{S\left[J\left(\underline{k}_{0}\right)-(J(0)+K(0))+J^{\prime}\left(\underline{k}_{0}\right)+\left(J^{\prime}(0)+K^{\prime}(0)\right)\right] \mathrm{m}-2 \tilde{B}(\pi / 2)\right\}^{\frac{1}{2}}$ $\left.x \quad 2 S J^{\prime}\left(\underline{k}_{0}\right) m\right\}^{\frac{3}{2}}$.

At the point $K$ in the Brillouin zone, $J^{\prime}(\underline{q}), K^{\prime}(\underline{q})$, $J^{\prime}\left(\underline{q}^{+} \underline{k}_{0}\right)$ and $J^{\prime}\left(\underline{q}-\underline{k}_{0}\right)$ are all zero. Hence the modes are degenerate at this point.

\subsection{The Conical Spiral}

This structure occurs in Ho and Er below 20K. The magnitude of the spiral wave vector $\underline{k}_{0}$ is $2 \pi /(5.5 \mathrm{c})$ and $2 \pi /(4.1 \mathrm{c})$ respectively and the cone angle $\theta$ at $20 \mathrm{~K}$ is approximately $79^{\circ}$ in Ho (Koehler et. al. 1966) and $28.5^{\circ}$ in Er (Cable et. al. 1965). It is also evident that the planar spiral of the last subsection will become a conical spiral when a magnetic field is applied along the c-axis.

As discussed in section 2.6, the spin-wave energies cannot be given in a closed form for general q's in this structure. However, if $\mathrm{q}$ is in either of the planes TKHA or KMLH of Fig. 1 then equation [2.37] may be used and the spin-wave energies are,

$$
\begin{aligned}
{[4.13 a] \mathrm{E}_{\underline{q}}^{(i)}=} & \frac{1}{2} S m \cos \theta\left[J\left(\underline{q}+\underline{k}_{0}\right)-J\left(\underline{q}-\underline{k}_{0}\right) \pm\left(J^{\prime}\left(\underline{q}^{+} \underline{k}_{0}\right)-J^{\prime}\left(\underline{q}_{-}-\underline{k}_{0}\right)\right)\right] \\
& +\frac{1}{2}\left(\mathrm{~F}_{1} F_{2}\right)^{\frac{1}{2}}
\end{aligned}
$$

$[4.13 b] \quad E_{-\underline{q}}^{(i)}=-\frac{1}{2} S m \cos \theta\left[J\left(\underline{q}+\underline{k}_{0}\right)-J\left(\underline{q}-\underline{k}_{0}\right) \pm\left(J^{\prime}\left(\underline{q}^{+} \underline{k}_{0}\right)-J^{\prime}\left(\underline{q}-\underline{k}_{0}\right)\right)\right]$

$$
+\frac{1}{2}\left(F_{1} F_{2}\right)^{\frac{1}{2}}
$$

where

$$
[4.14 a] F_{I}=\operatorname{Sm}\left[2 J\left(\underline{k}_{0}\right)+2 J^{\prime}\left(\underline{k}_{0}\right)-J\left(q+\underline{k}_{0}\right)-J\left(\underline{q}-\underline{k}_{0}\right) \pm\left(J \cdot\left(\underline{q}+\underline{k}_{0}\right)+J^{\prime}\left(\underline{q}-\underline{k}_{0}\right)\right)\right]
$$


$[4.14 b] F_{2}=4 S m \cos ^{2} \theta\left[J(0)+K(0)+J^{\prime}(0)+K^{\prime}(0)\right]-2 S m \cos 2 \theta\left[J\left(\underline{k}_{0}\right)+J^{\prime}\left(\underline{k}_{0}\right)\right]$

$$
\begin{aligned}
& -\operatorname{Sm} \cos ^{2} \theta\left[J\left(\underline{q}+\underline{k}_{0}\right)+J\left(\underline{q}-\underline{k}_{0}\right) \mp\left(J^{\prime}\left(\underline{q}+\underline{k}_{0}\right)+J^{\prime}\left(\underline{q}-\underline{k}_{0}\right)\right]\right. \\
& -2 \operatorname{Sm} \sin ^{2} \theta\left[J(\underline{q})+K(\underline{q}) \mp\left(J^{\prime}(\underline{q})+K^{\prime}(\underline{q})\right)\right] \\
& -4 \tilde{B}(\theta)+4 g \mu_{B} H_{C} \cos \theta .
\end{aligned}
$$

Here $\widetilde{B}(\theta)$ is given by [2.29] and $\mathrm{H}_{C}$ is the component of the magnetic field along the c-axis. The components of $\underline{H}$ in the hexagonal plane average to zero, and the hexagonal crystal field and magnetoelastic terms have been omitted as in the last subsection.

A closed expression also exists when $q$ lies in the plane TKM. Then the solution [2.38]-[2.39] is applicable and since in this case $\mathrm{q}$ is perpendicular to $\underline{k}_{0}$ one has $\mathrm{J}\left(\underline{q}+\underline{k}_{0}\right)=\mathrm{J}\left(\underline{q}-\underline{k}_{0}\right)$ and $J^{\prime}\left(\underline{q}+\underline{k}_{0}\right)=J^{\prime}\left(\underline{q}-\underline{k}_{0}\right)$.

Finally, if $q$ is along either of the lines $\Gamma K$ or $K M,[4.13]$ reduces to

$$
E_{q}^{(i)}=E_{-q}^{(i)}=\frac{1}{2}\left(F_{1} F_{2}\right)^{\frac{1}{2}}
$$

with slightly simplified expressions for $F_{1}$ and $F_{2}$. We note that at the point $K, J^{\prime}(\underline{q}), K^{\prime}(\underline{q}), J^{\prime}\left(\underline{q}+\underline{k}_{0}\right)$ and $J^{\prime}\left(\underline{q}-\underline{k}_{0}\right)$ are all zero. Hence the modes are degenerate at this point, as they are in the other structures. 


\section{APPENDIX A}

\section{STABILITY CONDITION AT FINITE TEMPERATURES}

At finite temperatures the condition for stable equilibrium is that the free energy is a minimum with respect to variation of the angles $\theta$ and $\phi_{i}$. If the $\mathrm{E}_{\mathrm{q}}^{(i)}$ are the temperature dependent spin wave energies determined either by the equations of motion method of the present paper or by the free energy variational principle (Brooks et. al. 1968), the density matrix for the magnetic spin system is

$$
\begin{aligned}
\rho & =z^{-1} \exp \left[-\beta \sum_{\underline{q}, i} E_{q}^{(i)} n_{q}^{(i)}\right] \\
z & =\operatorname{Tr}\left\{\exp \left[-\beta \sum_{\underline{q}, i} E_{\underline{q}}^{(i)} n_{q}^{(i)}\right]\right\}
\end{aligned}
$$

From this the approximate free energy $\langle x\rangle+k T<$ In $\rho>$ may be shown to be equal to

$$
E_{0}+k T \sum_{\underline{q}, i} \ln \left[1-\exp \left(-\beta E_{\underline{q}}^{(i)}\right)\right]
$$

where $E_{0}$ is the energy of the system at $T=0$. Thus, differentiating with respect to $\theta$, for example, one obtains the stability condition,

$$
\partial E_{0} / \partial \theta+\underset{\underline{q}, i}{\sum}\left(\partial E_{\underline{q}}^{(i)} / \partial \theta\right)<n_{\underline{q}}^{(i)}>=0
$$

$$
\left\langle\mathrm{n}_{\underline{\mathrm{q}}}^{(i)}\right\rangle=\left[\exp \left(\beta \mathrm{E}_{\mathrm{q}}^{(i)}\right)-1\right]^{-1}
$$


But since

[A6]

$$
\langle\mu\rangle=E_{0}+\sum_{q, i} E_{\underline{q}}^{(i)}\left\langle n_{\underline{q}}^{(i)}\right\rangle
$$

we interpret [A4] in the following way: the stability condition at $\mathrm{T}>0$ may be obtained by differentiating with respect to the angular variable only those terms in $\langle x\rangle$ which depend explicitly on that variable, ignoring in the differentiation the implicit angle dependence of thermal averages such as $\left\langle\mathrm{n}_{\mathrm{q}}^{(i)}\right\rangle$. In other words the terms involving $\partial<\mathrm{n}_{\underline{g}}^{(i)}>/ \partial \theta$ which arise from differentiating [A6] with respect to $\theta$ are exactly cancelled by the contribution from the entropy term.

In the present work $\langle\alpha\rangle$ is expressed in terms of thermal averages of the spin operators. The demonstration above makes it plausible to ignore the implicit angle dependence of such thermal averages in arriving at the condition for stable equilibrium. 


\section{APPENDIX B \\ RANDOM PHASE APPROXIMATION AND IINEARIZATION OF THE EQUATIONS OF MOTION}

In this appendix we elaborate on the details of the random phase approximation which enables one to express the temperature dependence in terms of powers of the reduced magnetization. The approximations involved in linearizing the equations of motion are also described in more detail.

Let us first consider the evaluation of thermal averages in connection with the stability conditions. For the operators $\widetilde{\mathrm{O}}_{20}$ ' $\widetilde{O}_{40}$ and $\widetilde{O}_{60}$ which involve powers of $\mathrm{s}_{i}^{z}$ the following relation was found to be useful:

$$
\left(s_{i}^{z}\right)^{n} \tilde{\sim}(-s)^{n}+(2 s)^{-1}\left[(1-s)^{n}-(-s)^{n}\right] s_{i}^{+} s_{i}^{-}
$$

This is easily derived from the Wortis expansion [2.5] using the spin commutation relations to place all $s_{i}^{+}$operators to the left of $\mathrm{s}_{i}^{-}$operators before dropping terms in more than three operators. For example, in the case of $\widetilde{O}_{20}$ this gives

$$
\left.\widetilde{O}_{20}\left(\underline{S}_{i}\right)\right\rangle=\left\langle\frac{1}{2}\left\{3 S_{i}^{z 2}-S(S+1)\right\}\right\rangle
$$

$$
\approx s\left(s-\frac{1}{2}\right)<\left\{1-\left(3 / 2 s^{2}\right) s_{i}^{+} s_{i}^{-}\right\}>
$$

$$
\begin{aligned}
& \approx s\left(s-\frac{1}{2}\right)\left\{1-\left(3 / 2 N s^{2}\right) \underset{q}{\left.\sum<s_{q}^{+} s_{q}^{-}>\right\}}\right. \\
& \approx s\left(s-\frac{1}{2}\right)\{1-3(1-m)\}
\end{aligned}
$$

$$
\approx s\left(s-\frac{1}{2}\right) m^{3}
$$


where in the last steps we used equation [2.12] and expressed the result as a power of $\mathrm{m}$ following the procedure of Callen and Callen (1966) and others. In addition to this being valid for $m$ close to unity, it also ensures that $\left\langle\mathrm{So}_{20}\right\rangle$ goes correctly to zero at the Curie temperature. In general we have,

$$
<\tilde{o}_{\ell 0}\left(s_{i}^{x}, s_{i}^{y}, s_{i}^{z}\right)>\approx s s\left(\frac{l-1}{2}\right) m^{\frac{l(l+1)}{2}}, l \geq 2
$$

where $S(n)$ is defined in [2.16].

A similar approach is taken in linearizing the equations of motion. For the crystal field and single-ion magnetoelastic terms, the commutators are most easily evaluated using equation [2.22]. Thus, for example, we obtain

$$
\left[S_{\underline{q}^{\prime}}^{-} \sum_{i} \widetilde{O}_{\ell m}\left(\underline{S}_{i}\right)\right]=[\ell(\ell+1)-m(m-1)]^{\frac{1}{2}} \mathrm{~N}^{\frac{-1}{2}} \sum_{i} \widetilde{o}_{\ell m-1}\left(\underline{S}_{i}\right) \exp \left(-i q \cdot \underline{r}_{i}\right)
$$

Substituting the first two terms of the wortis expansion for $\mathrm{s}_{i}^{z}$ and $\mathrm{T}_{i}^{z}$, or using $[B I]$ to express powers of $\mathrm{S}_{i}^{z}$ and $\mathrm{T}_{i}^{z}$, we find for $\ell=2$,

[B5a]

$$
\widetilde{\sigma}_{20}\left(\underline{s}_{i}\right)=\operatorname{s}(1 / 2)\left\{1-\left(3 / 2 s^{2}\right) s_{i}^{+} s_{i}^{-}\right\}
$$

$$
\widetilde{o}_{2 \pm 1}\left(\underline{s}_{i}\right)= \pm(3 / 2)^{\frac{1}{2}} \mathrm{~s}(1 / 2)\left\{s_{i}^{ \pm}-(2 s \mathrm{~s}(i / 2))^{-1} s_{i}^{+} s_{i}^{ \pm} s_{i}^{-}\right\}
$$

$[\mathrm{B} 5 \mathrm{C}]$

$$
\widetilde{O}_{2+2}\left(S_{i}\right)=(3 / 8)^{\frac{1}{2}} s_{i}^{ \pm} s_{i}^{ \pm}
$$

When these are inserted into [B4] and the spin operators are Fourier transformed, the following random phase approximations are 
used:

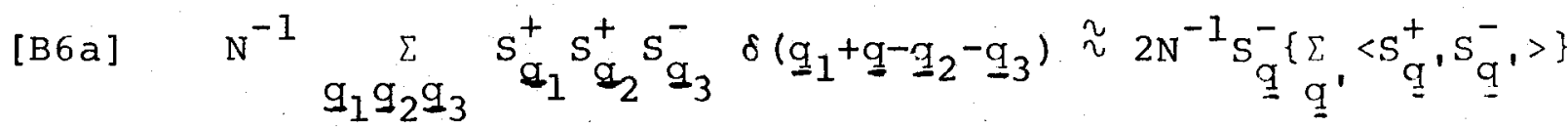

$$
+N^{-1} S_{-\underline{q}}^{+}\left\{\underline{q}^{\prime}<\underline{S}^{-}, \underline{S}^{-} \underline{\underline{q}}^{\prime}>\right\}
$$

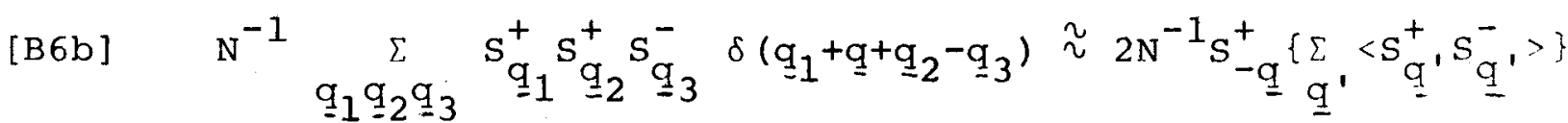

$$
+\mathrm{N}^{-1} \mathrm{~S}_{\underline{\mathrm{q}}^{-}}\left\{\underline{\Sigma}^{\prime}<\mathrm{S}_{\mathrm{q}^{\prime}}^{+} \mathrm{S}^{+} \underline{q}^{\prime}>\right\}
$$

The thermal averages $\Sigma<\mathrm{S}_{\mathrm{q}}^{+} \mathrm{S}^{+}>, \sum<\mathrm{T}_{\mathrm{q}}^{+} \mathrm{T}_{-\mathrm{q}^{+}}^{+}, \sum<\mathrm{S}_{\mathrm{q}}^{-} \mathrm{S}^{-} \mathrm{q}^{\mathrm{q}}>$ and $\sum<\mathrm{T}_{\mathrm{q}^{-}}^{-} \mathrm{T}^{-} \mathrm{q}^{-}$are

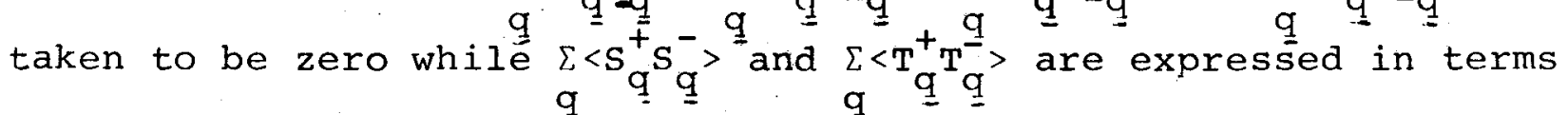
of the reduced magnetizätion by meañs of [2.12]. Hence the only non-zero commutators are

[B7b]

[B7C]

[B7d]

$$
\left[\mathrm{s}_{\underline{\underline{\mathrm{q}}}}^{-}, \sum_{i}^{\mathrm{L}} \widetilde{\mathrm{o}}_{20}\left(\underline{\mathrm{s}}_{\mathrm{i}}\right)\right]=-3 \mathrm{~s}(1 / 2) \mathrm{m}^{2} \mathrm{~s}_{\underline{\mathrm{q}}}^{-}
$$$$
\left[\mathrm{s}_{\underline{\mathrm{q}}}^{-}{ }_{\mathrm{i}} \widetilde{\mathrm{o}}_{22}\left(\underline{\mathrm{S}}_{\mathrm{i}}\right)\right]=\sqrt{6} \mathrm{~s}(1 / 2) \mathrm{m}^{2} \mathrm{~s}_{-\underline{\mathrm{q}}}^{+}
$$$$
\left[\mathrm{s}_{\underline{\underline{q}}}^{-}, \widetilde{\mathrm{O}}_{i 0}\left(\underline{\mathrm{S}}_{\mathrm{i}}\right)\right]=-10 \mathrm{~s}(3 / 2) \mathrm{m}^{9} \mathrm{~s}_{\underline{\mathrm{q}}}^{-}
$$

[B7e]

$$
\left.\left[\mathrm{s}_{\underline{\underline{i}}}^{-}, \sum_{42} \cdot \widetilde{\mathrm{o}}_{4}\right)\right]=3 \sqrt{10} \mathrm{~s}(3 / 2) \mathrm{m}^{9} \mathrm{~s}_{-\underline{q}}^{+}
$$

[B7f]

$$
\left[\underline{s}_{\underline{q}}^{-}, \Gamma_{i} \widetilde{O}_{60}\left(\underline{S}_{i}\right)\right]=-21 \mathrm{~s}(5 / 2) \mathrm{m}^{20} \mathrm{~s}_{\underline{q}}^{-}
$$

$$
\left[\mathrm{s}_{\underline{q}_{i}^{-}}^{-} \Gamma_{i} \widetilde{o}_{62}\left(\underline{s}_{i}\right)\right]=2 \sqrt{105} \mathrm{~s}(5 / 2) \mathrm{m}^{20} \mathrm{~s}_{-\underline{q}}^{+}
$$

with similar expressions for ${\mathrm{S}_{-q^{\prime}}^{+}}^{\mathrm{T}_{\mathrm{q}}^{-}}$and $\mathrm{T}_{-\underline{\underline{q}}}^{+}$. 
The following commutators are also non-zero, giving constant terms in the equations of motion:

[B 8 a]

$$
\left[\mathrm{s}_{\underline{q}}^{-}, \sum_{i} \widetilde{O}_{21}\left(\underline{S}_{i}\right)=\sqrt{6} \mathrm{~s} s(1 / 2) \mathrm{m}^{3} \sqrt{\mathrm{N}} \delta_{\underline{q}, 0}\right.
$$

$$
\left[\mathrm{s}_{\underline{q^{\prime}}}^{-} \sum_{i} \widetilde{\mathrm{O}}_{41}\left(\underline{S}_{i}\right)\right]=2 \sqrt{5} \mathrm{~s} \mathrm{~s}(3 / 2) \mathrm{m}^{10} \sqrt{\mathrm{N}} \delta_{\underline{q}, 0}
$$

[B 8C]

$$
\left[s_{q_{i}^{\prime}}^{-} \widetilde{o}_{61}\left(\underline{s}_{i}\right)\right]=\sqrt{42} \mathrm{~s} s(5 / 2) \mathrm{m}^{21} \sqrt{\mathrm{N}} \delta_{q^{\prime}, 0}
$$

However, when all these constant terms are grouped together, the stability conditions [2.15] and [2.20] cause them to vanish.

The two-ion terms in the Hamiltonian are best handled by first Fourier transforming them and then using the commutation relations of the spin-wave operators. The random phase approximation is then applied to [2.9] yielding the simple result.

$$
s_{\underline{q}}^{z}=-s m \delta_{\underline{q}, 0}
$$

Hence the equivalent commutation relations are

$$
\begin{aligned}
& {\left[s_{\underline{q}^{\prime}}^{+}, s_{\underline{q}^{\prime}}^{-}\right]=-2 s m \delta \underline{q}^{\prime}, \underline{q}} \\
& {\left[s_{q^{\prime}}^{z}, s_{q}^{ \pm},\right]= \pm N^{-1} s_{\underline{q}}^{ \pm}, \delta q, 0}
\end{aligned}
$$

which is the form used by Englert (1960). 
APPENDIX C

\section{TRANSFORMATION OF SPIN OPERATOR EQUIVALENTS}

In this appendix the relevant transformations are given for the crystal field and single-ion magnetoelastic terms. Using equation [2.3] the operator equivalents $\tilde{O}_{\ell m}$ can be rotated to equilibrium coordinates at each lattice site. However it is more convenient to write the results in terms of the linear combinations $\gamma_{l m}^{+}$and $\gamma_{l m}^{-}$defined in equation [3.4]. In general the transformations have the form, [Cla] $\gamma_{\ell m}^{+}\left(S_{i}^{\xi}, S_{i}^{n}, S_{i}^{\zeta}\right)=\frac{1}{2} \cos m \phi_{i} R_{l m}^{+}+\frac{i}{2} \sin m \phi_{i} R_{l m}^{-}$ [Clb] $\sigma_{\ell m}^{-}\left(S_{i}^{\xi}, S_{i}^{n}, S_{i}^{\zeta}\right)=-\frac{i}{2} \cos m \phi_{i} R_{\ell m}^{-}+\frac{1}{2} \sin m \phi_{i} R_{\ell m}^{+}$ where $R_{l_{m}}^{+}$and $R_{\ell m}^{-}$are functions of the cone angle $\theta$ and the equilibrium coordinates $s_{i}^{X}, s_{i}^{Y}, s_{i}^{z}$.

For the crystal field terms, only axial and hexagonal terms appear. The corresponding functions are,

$$
\begin{aligned}
\mathrm{R}_{20}^{+} & =2 \mathrm{P}_{2}^{0 \gamma_{20}}+\frac{2 \sqrt{6}}{3} \mathrm{P}_{2}^{1}\left(i \gamma_{21}^{-}\right)+\frac{\sqrt{6}}{3} \mathrm{P}_{2}^{2 \gamma_{22}^{+}} \\
\mathrm{R}_{40}^{+} & =2 \mathrm{P}_{4}^{0 \gamma_{40}}+\frac{2 \sqrt{5}}{5} \mathrm{P}_{4}^{1}\left(i \gamma_{41}^{-}\right)+\frac{\sqrt{10}}{15} \mathrm{P}_{4}^{2} \gamma_{42}^{+} \\
& +\frac{\sqrt{35}}{105} \mathrm{P}_{4}^{3}\left(i \tilde{O}_{43}^{-}\right)+\frac{\sqrt{70}}{420} \mathrm{P}_{4}^{4} \gamma_{44}^{+}
\end{aligned}
$$


69

[CH]

$$
\begin{aligned}
\mathrm{R}_{60}^{+} & =2 \mathrm{P}_{6}^{0 \tilde{O}_{60}}+\frac{2 \sqrt{42}}{21} \mathrm{P}_{6}^{1}\left(i \tilde{\gamma}_{61}^{-}\right)+\frac{\sqrt{105}}{105} \mathrm{P}_{6}^{2 \mathrm{O}_{62}^{+}} \\
& +\frac{\sqrt{105}}{630} \mathrm{P}_{6}^{3}\left(i \tilde{O}_{63}^{-}\right)+\frac{\sqrt{14}}{1260} \mathrm{P}_{6}^{4} \mathrm{O}_{64}^{+} \\
& +\frac{\sqrt{77}}{13860} \mathrm{P}_{6}^{5}\left(i \tilde{O}_{65}^{-}\right)+\frac{\sqrt{231}}{83160} \mathrm{P}_{6}^{6} \gamma_{66}^{+}
\end{aligned}
$$

[CF]

$$
\begin{aligned}
& R_{66}^{+}=\frac{\sqrt{231}}{16}\left\{\left(1-x^{2}\right)^{3} \gamma_{60}-\frac{2 \sqrt{42}}{7} \times\left(1-x^{2}\right)^{5 / 2}\left(i \gamma_{61}^{-}\right)\right. \\
& +\frac{\sqrt{105}}{7}\left(1-x^{2}\right)^{2}\left(1+x^{2}\right) \gamma_{62}^{+}-\frac{2 \sqrt{105}}{21}\left(1-x^{2}\right)^{3 / 2}\left(3 x+x^{3}\right)\left(i \gamma_{63}^{-}\right) \\
& +\frac{\sqrt{14}}{7}\left(1-x^{2}\right)\left(1+6 x^{2}+x^{4}\right) \tilde{o}_{64}^{+}-\frac{2 \sqrt{77}}{77}\left(1-x^{2}\right)^{\frac{1}{2}}\left(5 x+10 x^{3}+x^{5}\right)\left(i \delta_{65}^{-}\right) \\
& \left.+\frac{\sqrt{231}}{231}\left(1+15 x^{2}+15 x^{4}+x^{6}\right) \gamma_{66}^{+}\right\}
\end{aligned}
$$

where $P_{\ell}^{\mathrm{m}}$ is an associated Legendre function $\mathrm{P}_{Q}^{\mathrm{m}}(\cos 0)$ and $\mathrm{x}$ is equal to $\cos 0$. Note that $R_{\ell_{0}}^{-}$does not contribute to [Cl].

In Chapter 3, we considered magnetoelastic terms

only up to $\ell=4$. For the sake of completeness, the relevant functions up to $l=6$ are written down below.

[CF]

$$
\mathrm{R}_{21}^{+}=2 \mathrm{xO}_{21}^{+}+2\left(1-\mathrm{x}^{2}\right)^{\frac{1}{2}}\left(i_{22}^{\tilde{O}^{-}}\right)
$$


70

[C8] $\quad \mathrm{R}_{21}^{-}=-\sqrt{6} \mathrm{x}\left(1-\mathrm{x}^{2}\right)^{\frac{1}{2}} \gamma_{20}+2\left(2 \mathrm{x}^{2}-1\right)\left(i \gamma_{21}^{-}\right)+2 \mathrm{x}\left(1-\mathrm{x}^{2}\right)^{\frac{1}{2}} \gamma_{22}^{+}$

[c9] $R_{22}^{+}=\frac{\sqrt{6}}{2}\left(1-x^{2}\right) \tilde{\partial}_{20}-2 x\left(1-x^{2}\right)^{\frac{1}{2}}\left(i \gamma_{21}^{-}\right)+\left(1+x^{2}\right) \gamma_{22}^{+}$

$[\mathrm{Cl0}] \mathrm{R}_{22}^{-}=-2\left(1-\mathrm{x}^{2}\right)^{\frac{1}{2}} \tilde{\mathrm{O}}_{21}^{+}+2 \mathrm{x}\left(\mathrm{iO}_{22}^{-}\right)$

$[C 11] \mathrm{R}_{41}^{+}=\frac{1}{2} \mathrm{x}\left(7 \mathrm{x}^{2}-3\right) \gamma_{41}^{+}+\frac{\sqrt{2}}{2}\left(1-\mathrm{x}^{2}\right)^{\frac{1}{2}}\left(7 \mathrm{x}^{2}-1\left(\mathrm{i} \gamma_{42}^{-}\right)+\frac{\sqrt{7}}{2}\left(1-\mathrm{x}^{2}\right)(3 \mathrm{x}) \gamma_{43}^{+}\right.$

$+\frac{\sqrt{14}}{2}\left(1-x^{2}\right)^{3 / 2}\left(i \tilde{O}_{44}^{-}\right)$

[C12] $\mathrm{R}_{41}^{-}=\frac{\sqrt{5}}{2} \times\left(1-\mathrm{x}^{2}\right)^{\frac{1}{2}}\left(3-7 \mathrm{x}^{2}\right) \widetilde{\gamma}_{40}+\frac{1}{2}\left(3-27 \mathrm{x}^{2}+28 \mathrm{x}^{4}\right)\left(\mathrm{iO}_{41}^{-}\right)$

$+\sqrt{2}\left(1-x^{2}\right)^{\frac{1}{2}}\left(7 x^{3}-4 x\right) \gamma_{42}^{+}+\frac{\sqrt{7}}{2}\left(1-x^{2}\right)\left(4 x^{2}-1\right)\left(i \gamma_{43}^{-}\right)$

$+\frac{\sqrt{14}}{2} \times\left(1-x^{2}\right)^{3 / 2} z_{44}^{+}$

[C13] $\mathrm{R}_{42}^{+}=\frac{\sqrt{10}}{4}\left(1-\mathrm{x}^{2}\right)\left(7 \mathrm{x}^{2}-1\right) \tilde{\mathrm{O}}_{40}-\sqrt{2}\left(1-\mathrm{x}^{2}\right)^{\frac{1}{2}}\left(7 \mathrm{x}^{3}-4 \mathrm{x}\right)\left(\mathrm{i \tilde {O } _ { 4 1 } ^ { - }}\right)$

$+\left(1-6 x^{2}+7 x^{4}\right) \tilde{O}_{42}^{+}+\frac{\sqrt{14}}{2}\left(1-x^{2}\right)^{\frac{1}{2}}\left(2 x^{3}\right)\left(i \tilde{O}_{43}\right)$

$+\frac{\sqrt{7}}{2}\left(1-x^{2}\right)\left(1+x^{2}\right) \tilde{O}_{44}^{+}$

[C14] $\mathrm{R}_{42}^{-}=-\frac{\sqrt{2}}{2}\left(1-\mathrm{x}^{2}\right)^{\frac{1}{2}}\left(7 \mathrm{x}^{2}-1\right) \gamma_{41}^{+}+\left(7 \mathrm{x}^{3}-5 \mathrm{x}\right)\left(i \gamma_{42}^{-}\right)$

$+\frac{\sqrt{14}}{2}\left(1-x^{2}\right)^{\frac{3}{2}}\left(3 x^{2}-1\right) \tilde{o}_{43}^{+}+\frac{\sqrt{7}}{2}\left(1-x^{2}\right)(2 x)\left(i \gamma_{44}^{-}\right)$ 
71

$$
\begin{aligned}
& \text { [C15] } \mathrm{R}_{44}^{+}=\frac{\sqrt{70}}{8}\left(1-\mathrm{x}^{2}\right)^{2} \mathrm{O}_{20}-\frac{\sqrt{14}}{2}\left(1-\mathrm{x}^{2}\right)^{3 / 2} \times\left(\mathrm{iO}_{41}^{-}\right) \\
& +\frac{\sqrt{7}}{2}\left(1-x^{2}\right)\left(1+x^{2}\right) \tilde{o}_{42}^{+} \\
& -\frac{\sqrt{2}}{2}\left(1-x^{2}\right)^{\frac{1}{2}}\left(3 x+x^{3}\right)\left(i \tilde{O}_{43}^{-}\right)+\frac{1}{4}\left(1+6 x^{2}+x^{4}\right) \tilde{O}_{44}^{+} \\
& {[\mathrm{Cl6}] \mathrm{R}_{44}^{-}=-\frac{\sqrt{14}}{2}\left(1-\mathrm{x}^{2}\right)^{3 / 2 \tilde{\mathrm{O}}_{41}^{+}}+\sqrt{7}\left(1-\mathrm{x}^{2}\right) \times\left(\mathrm{i} \tilde{\gamma}_{42}^{-}\right)} \\
& -\frac{\sqrt{2}}{2}\left(1-x^{2}\right)^{\frac{1}{2}}\left(1+3 x^{2}\right) \gamma_{43}^{+}+\frac{1}{4}\left(4 x+4 x^{3}\right)\left(i \gamma_{44}^{-}\right) \\
& \text {[C17] } R_{61}^{+}=\frac{1}{4}\left(33 x^{5}-30 x^{3}+5 x\right) \gamma_{61}^{+}+\frac{\sqrt{10}}{40}\left(1-x^{2}\right)^{\frac{1}{2}}\left(165 x^{4}-90 x^{2}+5\right)\left(i \gamma_{62}^{-}\right) \\
& +\frac{3 \sqrt{10}}{8}\left(1-x^{2}\right)\left(11 x^{3}-3 x\right) \tilde{o}_{63}^{+}+\frac{\sqrt{3}}{4}\left(1-x^{2}\right)^{3 / 2}\left(22 x^{2}-2\right)\left(i 0_{64}^{-}\right) \\
& +\frac{\sqrt{66}}{8}\left(1-x^{2}\right)^{2}(5 x) \tilde{\sigma}_{65}^{+}+\frac{3 \sqrt{22}}{8}\left(1-x^{2}\right)^{5 / 2}\left(i \gamma_{66}^{-}\right) \\
& {[C 18] \mathrm{R}_{61}^{-}=-\frac{\sqrt{42}}{21} \mathrm{P}_{6}^{1} \widetilde{\sigma}_{60}+\frac{1}{4}\left(198 \mathrm{x}^{6}-285 \mathrm{x}^{4}+100 \mathrm{x}^{2}-5\right)\left(i \gamma_{61}^{-}\right)} \\
& +\frac{\sqrt{10}}{8}\left(1-x^{2}\right)^{\frac{1}{2}}\left(99 x^{5}-102 x^{3}+19 x\right) \tilde{0}_{62}^{+} \\
& +\frac{3 \sqrt{10}}{8}\left(1-x^{2}\right)\left(22 x^{4}-15 x^{2}+1\right) \cdot\left(i \tilde{O}_{63}^{-}\right) \\
& +\frac{\sqrt{3}}{4}\left(1-x^{2}\right)^{3 / 2}\left(33 x^{3}-13 x\right) \gamma_{64}^{+} \\
& +\frac{\sqrt{66}}{8}\left(1-x^{2}\right)^{2}\left(6 x^{2}-1\right)\left(i \widetilde{\gamma}_{65}^{-}\right)+\frac{3 \sqrt{22}}{8}\left(1-x^{2}\right)^{5 / 2} x_{66}^{+}
\end{aligned}
$$


72

[C19] $\mathrm{R}_{62}^{+}=\frac{\sqrt{105}}{16}\left(1-\mathrm{x}^{2}\right)\left(33 \mathrm{x}^{4}-18 \mathrm{x}^{2}+1\right) \tilde{O}_{60}$

$$
\begin{aligned}
& -\frac{\sqrt{10}}{8}\left(1-x^{2}\right)^{\frac{1}{2}}\left(99 x^{5}-102 x^{3}+19 x\right)\left(i \gamma_{61}^{-}\right) \\
& +\frac{1}{16}\left(495 x^{6}-735 x^{4}+289 x^{2}-17\right) \tilde{O}_{62}^{+} \\
& +\frac{3}{8}\left(1-x^{2}\right)^{\frac{1}{2}}\left(55 x^{5}-50 x^{3}+11 x\right)\left(i \tilde{0}_{63}^{-}\right) \\
& +\frac{\sqrt{30}}{16}\left(1-x^{2}\right)\left(33 x^{4}-10 x^{2}+1\right) \tilde{\sigma}_{64}^{+} \\
& +\frac{\sqrt{165}}{8}\left(1-x^{2}\right)^{3 / 2}\left(3 x^{3}+x\right)\left(i \tilde{\sigma}_{65}^{-}\right)+\frac{3 \sqrt{55}}{16}\left(1-x^{2}\right)^{2}\left(1+x^{2}\right) \tilde{o}_{66}^{+}
\end{aligned}
$$

[C20] $\mathrm{R}_{62}^{-}=-\frac{\sqrt{10}}{8}\left(1-\mathrm{x}^{2}\right)^{\frac{1}{2}}\left(33 \mathrm{x}^{4}-18 \mathrm{x}^{2}+1\right) \mathrm{O}_{61}^{+}$

$$
\begin{aligned}
& +\frac{1}{16}\left(330 x^{5}-372 x^{3}+74 x\right)\left(i \gamma_{62}^{-}\right)+\frac{3}{8}\left(1-x^{2}\right)^{\frac{1}{2}}\left(55 x^{4}-42 x^{2}+3\right) \gamma_{63}^{+} \\
& +\frac{\sqrt{30}}{16}\left(1-x^{2}\right)\left(44 x^{3}-20 x\right)\left(i \gamma_{64}^{-}\right) \\
& +\frac{\sqrt{165}}{8}\left(1-x^{2}\right)^{3 / 2}\left(5 x^{2}-1\right) \tilde{o}_{65}^{+}+\frac{3 \sqrt{55}}{16}\left(1-x^{2}\right)^{2}(2 x)\left(i \gamma_{66}^{-}\right)
\end{aligned}
$$

$$
\text { [C21] } \begin{aligned}
\mathrm{R}_{64}^{+} & =\frac{3 \sqrt{14}}{16}\left(1-\mathrm{x}^{2}\right)^{2}\left(11 \mathrm{x}^{2}-1\right) \gamma_{60}-\frac{\sqrt{3}}{4}\left(1-\mathrm{x}^{2}\right)^{3 / 2}\left(33 \mathrm{x}^{3}-13 x\right)\left(i \gamma_{61}^{-}\right) \\
& +\frac{\sqrt{30}}{16}\left(1-\mathrm{x}^{2}\right)\left(33 \mathrm{x}^{4}-10 \mathrm{x}^{2}+1\right) \gamma_{62}^{+} \\
& -\frac{\sqrt{30}}{8}\left(1-\mathrm{x}^{2}\right)^{\frac{1}{2}}\left(11 \mathrm{x}^{5}+2 \mathrm{x}^{3}-5 \mathrm{x}\right)\left(i \gamma_{63}^{-}\right)
\end{aligned}
$$


73

$$
\begin{aligned}
& +\frac{1}{8}\left(33 x^{6}+35 x^{4}-65 x^{2}+13\right) z_{64}^{+} \\
& +\frac{\sqrt{22}}{8}\left(1-x^{2}\right)^{\frac{1}{2}}\left(3 x^{5}+10 x^{3}-5 x\right)\left(i \gamma_{65}^{-}\right) \\
& +\frac{\sqrt{66}}{16}\left(1-x^{2}\right)\left(1+6 x^{2}+x^{4}\right) \tilde{o}_{66}^{+}
\end{aligned}
$$

[C22]

$$
\begin{aligned}
R_{64}^{-} & =-\frac{\sqrt{3}}{4}\left(1-x^{2}\right)^{3 / 2}\left(22 x^{2}-2\right) \gamma_{61}^{+} \\
& +\frac{\sqrt{30}}{16}\left(1-x^{2}\right)\left(44 x^{3}-20 x\right)\left(i \tilde{O}_{62}^{-}\right) \\
& -\frac{\sqrt{30}}{8}\left(1-x^{2}\right)^{\frac{1}{2}}\left(22 x^{4}-16 x^{2}+2\right) \gamma_{63}^{+} \\
& +\frac{1}{8}\left(88 x^{5}-80 x^{3}+8 x\right)\left(i \tilde{O}_{64}^{-}\right)+\frac{\sqrt{2} \overline{2}}{8}\left(1-x^{2}\right)^{\frac{1}{2}}\left(10 x^{4}-2\right) \tilde{O}_{65}^{+} \\
& +\frac{\sqrt{66}}{16}\left(1-x^{2}\right)\left(4 x+4 x^{3}\right)\left(i \gamma_{66}^{-}\right)
\end{aligned}
$$

$$
\begin{aligned}
& \text { [C23] } R_{65}^{+}=\frac{\sqrt{66}}{8}\left(1-x^{2}\right)^{2}(5 x) \tilde{o}_{61}^{+}-\frac{\sqrt{165}}{8}\left(1-x^{2}\right)^{3 / 2}\left(5 x^{2}-1\right)\left(i \tilde{O}_{62}^{-}\right) \\
& +\frac{\sqrt{165}}{8}\left(1-x^{2}\right)\left(5 x^{3}-x\right) \tilde{\sigma}_{63}^{+}-\frac{\sqrt{22}}{8}\left(1-x^{2}\right)^{\frac{1}{2}}\left(10 x^{4}-2\right)\left(i \tilde{O}_{64}^{-}\right) \\
& +\frac{1}{8}\left(25 x^{5}+10 x^{3}-19 x\right) \gamma_{65}^{+}+\frac{\sqrt{3}}{8}\left(1-x^{2}\right)^{\frac{1}{2}}\left(1+10 x^{2}+5 x^{4}\right)\left(\text { iO }_{66}^{-}\right)
\end{aligned}
$$

$$
\begin{aligned}
\text { [C24] } \mathrm{R}_{65}^{-} & =-\frac{3 \sqrt{77}}{8}\left(1-\mathrm{x}^{2}\right)^{5 / 2} \mathrm{xO}_{60}+\frac{\sqrt{66}}{8}\left(1-\mathrm{x}^{2}\right)^{2}\left(6 \mathrm{x}^{2}-1\right)\left(i \tilde{\sigma}_{61}^{-}\right) \\
& -\frac{\sqrt{165}}{8}\left(1-\mathrm{x}^{2}\right)^{3 / 2}\left(3 \mathrm{x}^{3}+\mathrm{x}\right) \mathrm{\gamma}_{62}^{+}
\end{aligned}
$$




$$
\begin{aligned}
& +\frac{\sqrt{165}}{8}\left(1-x^{2}\right)\left(2 x^{4}+3 x^{2}-1\right)\left(i \gamma_{63}^{-}\right) \\
& -\frac{\sqrt{22}}{8}\left(1-x^{2}\right)^{\frac{1}{2}}\left(3 x^{5}+10 x^{3}-5 x\right) \gamma_{64}^{+} \\
& +\frac{1}{8}\left(6 x^{6}+35 x^{4}-20 x^{2}-5\right)\left(i \tilde{O}_{65}^{-}\right) \\
& +\frac{\sqrt{3}}{8}\left(1-x^{2}\right)^{\frac{1}{2}}\left(5 x+10 x^{3}+x^{5}\right) \gamma_{66}^{+} .
\end{aligned}
$$

Note that the functions $R_{43}^{ \pm}$and $R_{63}^{ \pm}$are not included for the reasons stated in section 3.1 . 
REFERENCES

Bar'yakhtar, V.G. and Maleev, S.V. 1963. Fiz. Tverdoga Tela

5, 1175. (English Translation, Soviet Physics - Solid

State $\underline{5}, 858$.

Birgeneau, R.J. 1967. Can. J. Phys. $\underline{45}, 3761$.

Birss, R.R. 1964. Symmetry and Magnetism (North-Holland Publishing Company, Amsterdam).

Brooks, M.S.S., Goodings, D.A. and Ralph, H.I. 1968. J. Phys. C. (Solid st. Phys.) Ser. 2, 1, 132 .

Brooks, M.S.S. and Goodings, D.A. 1968. J. Phys. C. (Solid St. Phys.) Ser. 2, 1, 1279 .

Brooks, M.S.S. 1969. J. Phys. C. (Solid St. Phys.) Ser. 2, 2, 1016.

Brooks, M.S.S. 1970. Phys. Rev. Bl, 2257.

Buckmaster, H.A. 1962. Can. J. Phys. 40, 1670.

Cable, J.W., Wollan, E.O., Koehler, W.C. and Wilkinson, M.K.

1965. Phys. Rev. 140, A1896.

Callen, E. and Callen, H.B. 1965. Phys. Rev. 139, A455.

Callen H.B. and Callen E. 1966. J. Phys. Chem. Solids $27,1271$.

Clark, A.E., DeSavage, B.F. and Bozorth, R. 1965. Phys. Rev. $138, \mathrm{~A} 216$.

Cooper, B.R., Elliott, R.J., Nettel, S.J. and Suhl, H. 1962. Phys. Rev. 127, 57 .

Cooper, B.R. and Elliott, R.J. 1963. Phys. Rev. 131, 1043.

Cooper, B.R. 1968a. Phys. Rev. 169, 281.

Cooper, B.R. 1968b. Solid State Phys. 21, 393.

Edmonds, A.R. 1957. Angular Momentum in Quantum Mechanics (Princeton University Press). 
Elliott, R.J. 1961. Phys. Rev. 124, 346.

Elliott, R.J. and Lange, R.V. 1966. Phys. Rev. 152, 235.

Englert, F. 1960. Phys. Rev. Lett. $\underline{5}, 102$.

Enz, U. 1960. Physica 26, 698 .

Ginzburg, V.L. and Fain, V.M. 1961. Soviet Phys. JETP 12, 923.

Hutchings, M.T. 1964. Solid State Phys: 16, 227.

Jordan, R.G. and Lee, E.W. 1967. Proc. Phys. Soc. 92, 1074.

Kaplan, T.A. 1961. Phys. Rev. 124, 329.

Kasuya, T. 1956. Progr. Theoret. Phys. 16, 45.

Keffer, F. 1966. Handbuch der Physik (Edited by S. Flügge, Springer-Verlag, Berlin) Vol. XVIII/2.

Kittel, C. 1948. Phys. Rev. 73, 155.

Kittel, C. and Van Vleck, J.H. 1960. Phys. Rev. 118, 1231.

Kittel, C. 1968. Solid State Phys. 21, 1.

Koehler, W.C., Cable, J.W., Wilkinson, M.K. and Wollan E.O. 1966.

Phys. Rev. 151, 414 .

Lindgård, P.A., Kowalska, A. and Laut, P. 1967. J. Phys. Chem. Solids $\underline{28}, 1357$.

Lindgärd, P.A. 1971. to be published. Mason, W.P. 1954. Phys. Rev. 96, 302. Nagamiya, T. 1967. Solid State Phys. 20, 305. Nielsen, M., Bjerrum Mфller, H., Lindgărd, P.A. and Mackintosh, A.R. 1970. Phys. Rev. Lett. 25, 1451.

Orbach, R. 1961. Proc. Roy. Soc. A264, 458.

Rhyne, J.J. and Legvold, S. 1965. Phys. Rev. 138, A507. Rhyne, J.J. and Clark, A.E. 1967. J. Appl. Phys. 38, 1379. 
Ruderman, M.A. and Kittel, C. 1954. Phys. Rev. 96, 99.

Smith, D. and Thornley, J.H.M. 1966. Proc. Phys. Soc. 89, 779. Stevens, K.W.H. 1952. Proc. Phys. Soc. A65, 209.

Turov, E.A. and Shavrov, V.G. 1965. Soviet Phys. - Solid State 7. 166 .

Wortis, M. 1959. Ph.D. Thesis, Harvard University.

Yosida, K. 1.957. Phys. Rev. 106, 893.

Yosida, K. and Miwa, H. 1961. J. App1. Phys. (Supp1.) 32, 8S. Yosida, K. and Watabe, A. 1962. Progr. Theoret. Phys. (Kyoto) $\underline{28}, 361$. 